\title{
SCIENTIFIC REPORTS

\section{OPEN Whole Genome Sequencing of the Mutamouse Model Reveals Strain- and Colony-Level Variation, and Genomic Features of the Transgene Integration Site}

Received: 20 December 2018

Accepted: 5 September 2019

Published online: 24 September 2019

\author{
Matthew J. Meier $\mathbb{C}^{1,2}$, Marc A. Beal $\mathbb{C}^{1,3}$, Andrew Schoenrock ${ }^{1}$, Carole L. Yauk ${ }^{1}$ \& \\ Francesco Marchetti $\mathbb{D}^{1}$
}

The MutaMouse transgenic rodent model is widely used for assessing in vivo mutagenicity. Here, we report the characterization of MutaMouse's whole genome sequence and its genetic variants compared to the $\mathrm{C} 57 \mathrm{BL} / 6$ reference genome. High coverage ( $>50 \mathrm{X}$ ) next-generation sequencing (NGS) of whole genomes from multiple MutaMouse animals from the Health Canada (HC) colony showed $\sim 5$ million SNVs per genome, $\sim 20 \%$ of which are putatively novel. Sequencing of two animals from a geographically separated colony at Covance indicated that, over the course of 23 years, each colony accumulated 47,847 (HC) and 17,677 (Covance) non-parental homozygous single nucleotide variants. We found no novel nonsense or missense mutations that impair the MutaMouse response to genotoxic agents. Pairing sequencing data with array comparative genomic hybridization $(\mathrm{aCGH})$ improved the accuracy and resolution of copy number variants (CNVs) calls and identified 300 genomic regions with CNVs. We also used long-read sequence technology (PacBio) to show that the transgene integration site involved a large deletion event with multiple inversions and rearrangements near a retrotransposon. The MutaMouse genome gives important genetic context to studies using this model, offers insight on the mechanisms of structural variant formation, and contributes a framework to analyze aCGH results alongside NGS data.

Mutagenicity assessment of chemicals and pharmaceuticals prior to commercial use is essential to the protection of public health and forms the primary component of human health risk assessment. The majority of mutagenicity assessment has been undertaken in genetically modified Salmonella bacteria (i.e., the Ames assay) providing a first-tier assay in conventional genotoxicity testing paradigms ${ }^{1}$. However, mutagenicity in non-metabolically competent bacterial lines, for many reasons, may not be recapitulated in vivo and reflect potential hazards for humans. Thus, most paradigms require follow-up testing in an in vivo model $^{2}$ and the laboratory mouse has been an indispensable tool for understanding hazards posed by environmental chemicals and pharmaceuticals. Beginning in the late 1980s, transgenic rodent (TGR) mutation reporter models ${ }^{3}$ provided an unprecedented tool for quantifying mutations in mammalian cells. Since this time, TGR models have become the basis of in vivo mutagenicity assessment, and the use of TGR models to evaluate the safety of chemicals is described in the Organisation for Economic Co-operation and Development test guideline $488^{4}$.

The MutaMouse transgenic model is the most widely used TGR system in regulatory genetic toxicology ${ }^{3,5}$. The MutaMouse contains a recoverable lacZ transgene that can be isolated from the DNA of any tissue and used to score mutations with an in vitro positive selection assay, ${ }^{6,7}$. The TGR assay provides unique opportunities to study mutations in any tissue. In addition, studies can be paired with next-generation sequencing to reveal details of changes in mutation spectrum to inform on the mechanism of action of the test agent ${ }^{8,9}$. Over 130 chemical and

${ }^{1}$ Environmental Health Science and Research Bureau, Health Canada, Ottawa, ON, Canada. ${ }^{2}$ Present address: Ecotoxicology and Wildlife Health Division, Environment and Climate Change Canada, Ottawa, ON, Canada. ${ }^{3}$ Present address: Existing Substances Risk Assessment Bureau, Health Canada, Ottawa, ON, Canada. Correspondence and requests for materials should be addressed to F.M. (email: francesco.marchetti@canada.ca) 
physical agents have been tested with the MutaMouse model (representing over $50 \%$ of the 250 agents that have been tested in total) ${ }^{3}$. Given the importance of mutagenicity assessment and the prevalent use of the MutaMouse in such evaluations, the availability of a MutaMouse reference genome can provide a deeper understanding of its genetic traits that may be relevant to mutagenicity, chemical metabolism, and toxicology in general.

Analyses of gene expression profiles and other biochemical changes (e.g., enzyme activity) in response to chemicals exposure are increasingly used to complement genetic toxicology data in MutaMouse models ${ }^{10-13}$. Rapid advances in next-generation sequencing (NGS) technology have allowed the application of whole-genome approaches to understanding fundamental mechanisms of mutagenesis ${ }^{14-17}$. Fully annotated and characterized genomes of TGR models can improve the utility of these strains for genetic toxicology research by increasing the understanding of the potential biological consequences of genetic variants, and facilitating integration with modern genomic methods.

The high-quality genome assembly of the C57BL/6 mouse comprises the reference sequence against which all newly sequenced mouse genomes are compared ${ }^{18}$. The first studies using NGS to characterize mouse genomes ${ }^{19-25}$ established some important approaches to determine copy number variants ${ }^{19,24}$, genetic variation with respect to phenotype $\mathrm{e}^{23}$ and structural variation among 17 mouse strains $\mathrm{s}^{20,22}$. New methods for copy number variant $(\mathrm{CNV})$ and structural variant (SV) discovery have been developed ${ }^{16}$ and analyses of repetitive elements on a genome-wide scale have improved our understanding of the role that this type of variation plays in disease ${ }^{26}$. Comparative transcriptomic studies have provided an initial survey of the molecular basis of phenotypic variation between inbred mouse strains ${ }^{27}$. Recently, the whole genome of C57BL/6J Eve, the female mouse from which the current $\mathrm{C} 57 \mathrm{BL} / 6 \mathrm{~J}$ mice descend, has been reported and identified genetic variation that most closely represent recent generations of $\mathrm{C} 57 \mathrm{BL} / 6 \mathrm{~J}$ mice than the original genome ${ }^{28}$.

The Mouse Genomes Project (http://www.sanger.ac.uk/science/data/mouse-genomes-project) has sequence data and variation for 36 fully sequenced strains as of 2019, while 16 of those also have completed de novo assemblies and gene annotation files ${ }^{25}$. Furthermore, the Mouse Genome Informatics database (MGI; http://www. informatics.jax.org/) provides access to a wide range of resources to facilitate studies on human health and disease $^{29}$ to provide context to whole genome sequence data and integrate other data types and analytical tools (e.g., pathways, gene expression, phenotypes, etc.). This work has driven forward the field of comparative genomics in mice $^{30,31}$. Finally, quantitative trait loci have become an important resource for linking genotype to phenotype ${ }^{32}$ and deep sequencing of multiple inbred mouse strains has been performed for such purposes ${ }^{33}$.

Here, we sequenced multiple MutaMouse animals with deep sequencing ( $>50 \mathrm{X})$ and multiple platforms to obtain high-quality variant calls. We used array comparative genomic hybridization (aCGH) in parallel with NGS data to characterize putative CNVs. Finally, we compared the genetic variation between two geographically separated colonies of MutaMouse mice, to define the genetic variation existing within and across MutaMouse colonies, divergence from parental strains, and identify functional mutations. We also conducted the first characterization of the breakpoints at the transgene integration site.

\section{Results}

Sequence data. Seven MutaMouse animals, including five males obtained from a breeding colony maintained at Health Canada (HC) in Ottawa, Canada, and one male and one female obtained from a breeding colony at Covance, UK, were sequenced in this study. Sequencing on the Illumina HiSeq and NextSeq platforms together produced between 54 to $80 \mathrm{X}$ median genome-wide read depth for the five males from the HC colony. The NextSeq coverage for the animals from the Covance colony was 28-30X. Finally, the Pacific Biosciences reads from MutaMouse animal 2 aligned with a median depth of 3.5x. Mean fragment length, total raw basepairs, and median depth of aligned reads for all animals are shown in Table 1.

Single nucleotide variants and inheritance from parental mouse strains DBA/2J and BALB/ CJ. We predicted single nucleotide variants (SNVs) and short insertions or deletions (indels) using the HaplotypeCaller from GATK, relative to the mm10 reference genome (Genome Reference Consortium Mouse Build 38). Raw variants were filtered using variant quality score recalibration with a sensitivity of $99.9 \%$. In total, the GATK workflow predicted between 4,949,501 to 5,161,277 variants for each animal (Fig. 1A; Supplemental Table S1). The majority of variants were SNVs (78-80\%), and most (80-84\%) were already present in dbSNP v142. We found that many of these SNVs (38\% of MutaMouse SNVs) were present in both parental strains (Fig. 1A), and some variants were inherited only from BALB/cJ (13\%) or DBA/2J (18\%); in total, $69 \%$ of all MutaMouse SNVs appear to originate from a parental strain. Here, we define BALB/cJ and DBA/2J as the parental strains, although the MutaMouse was created from the mating of two [BALB/cJ X DBA/2J]F 1 hybrids. It is likely that random assortment during the creation of the MutaMouse strain resulted in a slight bias toward DBA/2J chromosomal segments in the final strain 40.6. In particular, there is an obvious lack of BALB/cJ sequences in the $\mathrm{p}$ arms of chromosomes 2 and X (Fig. 2). Of the remaining fraction of MutaMouse SNVs (31\%) not found in parental strains, $11 \%$ (i.e., approximately $1 / 3$ of the non-parental MutaMouse SNVs) are found in dbSNP. This $11 \%$ portion could represent: (1) false negatives in the BALB/cJ or DBA/2J reference sequences used; (2) variants that are found in $\mathrm{BALB} / \mathrm{c}$ or DBA/2J but not in those animals sequenced by the mouse genomes project; and, (3) false positives resulting from systematic or bioinformatic errors that are in both dbSNP and the MutaMouse data. The remaining $20 \%$ of variants (i.e., approx. 937,000-989,000, depending on the animal; Supplemental Table S1) identified in this study are putatively private MutaMouse variants that arose during the over 25 years of breeding at HC.

Structural and copy number variants. Both aCGH and NGS data were used to identify SVs and CNVs. There were $300 \mathrm{CNV}$ s called using R-Gada from the aCGH probe values, of which 297 could be converted to mm10 coordinates. NGS data was analyzed using two methodologies: Manta and CNVnator. Manta was used to 


\begin{tabular}{|c|c|c|c|c|c|}
\hline \multicolumn{2}{|l|}{ Animal } & \multirow{2}{*}{$\begin{array}{l}\text { Platform and library type } \\
\text { Illumina TruSeq }^{\text {a }}\end{array}$} & \multirow{2}{*}{$\begin{array}{l}\text { Mean fragment length (bp) } \\
480\end{array}$} & \multirow{2}{*}{$\begin{array}{l}\text { Total raw bp } \\
160,972,859,100\end{array}$} & \multirow{2}{*}{\begin{tabular}{|l|}
$\begin{array}{l}\text { Median depth } \\
\text { of aligned reads }\end{array}$ \\
$55 \mathrm{X}$ \\
\end{tabular}} \\
\hline \multirow{6}{*}{$\begin{array}{l}\text { Health Canada } \\
\text { MutaMouse }\end{array}$} & 1 & & & & \\
\hline & \multirow[t]{2}{*}{2} & $\begin{array}{l}\text { Illumina TruSeq and Nextera } \\
\text { Mate Pair }\end{array}$ & $\begin{array}{l}\text { 3,078 (Nextera Mate Pair) } \\
480 \text { (TruSeq) }\end{array}$ & $270,924,369,600$ & $81 \mathrm{X}$ \\
\hline & & PacBio RSII ${ }^{\mathrm{b}}$ & 20,000 & $11,900,000,000$ & $3.5 \mathrm{X}$ \\
\hline & 3 & Illumina TruSeq & 450 & $159,512,702,850$ & $55 \mathrm{X}$ \\
\hline & 4 & Illumina TruSeq & 450 & $159,691,600,650$ & $54 \mathrm{X}$ \\
\hline & 5 & Illumina TruSeq & 444 & $196,932,569,400$ & $71 \mathrm{X}$ \\
\hline \multirow{2}{*}{ Covance MutaMouse } & Male & Illumina Nextera Mate Pair & 3,849 (Nextera mate pair) & $144,615,033,150$ & $32 \mathrm{X}$ \\
\hline & Female & Illumina Nextera Mate Pair & 3,414 (Nextera mate pair) & $131,111,604,600$ & $28 \mathrm{X}$ \\
\hline
\end{tabular}

Table 1. Animals sequenced in this study and library/coverage statistics. ${ }^{\mathrm{a} L i b r a r i e s ~ b u i l t ~ b y ~ G e ́ n o m e ~}$

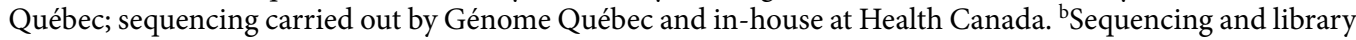
construction performed by Génome Québec.

call putative SVs (translocations, tandem duplications, deletions, and insertions) from Illumina NGS data based on discordant read pairs and local de novo assembly (Figs 1B, 2; Supplemental Table S1). Each MutaMouse animal had an average of approximately 17,000 deletions, 7,000 insertions, 1,100 tandem duplications, 1,000 inversions, and 7,000 translocation breakpoints. When considering only SV calls that were common to all animals, there were 4,795 deletions, 1,961 insertions, 190 tandem duplications, 239 inversions, and 686 translocation breakpoints. CNVnator, which predicts CNVs from NGS data based on read depth, identified an average of approximately 2,500 deletions and 1,800 duplications per animal.

Because SVs calls are less reliable than SNV calls, we limited our dataset to predicted variants found in all five animals sequenced to enrich for robust predictions and determined the overlap in predicted CNVs among aCGH, CNVnator, and Manta (Fig. 1C). In total, 64 events were predicted by all three methods (Fig. 1C). This number mainly reflects the larger CNVs that were predicted confidently by aCGH. However, each method produced a large number of predicted SVs that are exclusive to that dataset. Because our analysis included only SVs that were found in all five animals, many spurious calls were eliminated; thus, the significant differences between call sets produced by aCGH, CNVnator, and Manta can be attributed largely to analytical differences between the methods rather than false positives. Overall, these methods provide a picture of the genome that encompasses many different SV types across different scales.

Biological consequences of genetic variants. We used the Ensembl Variant Effect Predictor (VEP) and SnpEff to characterize the predicted biological effects of the SNVs and small indels identified through sequencing. Most of these variants fall into the category of intergenic or intronic (average of 3.2 million and 1.9 million, respectively; Fig. 3). Many variants also fall within $5 \mathrm{~kb}$ upstream or downstream of genes (average of 0.25 million each). Comparatively few variants fall within exons $(\sim 35,000), 3^{\prime}$ UTRs $(\sim 33,000), 5^{\prime}$ UTRs $(\sim 5,400)$, splice sites $(\sim 3,700)$, or otherwise influence transcription $(\sim 12)$. Of variants that fall within exons, an average of 10,045 missense variants were observed in each mouse $(9,520-10,558)$, while synonymous variants representing between 15,833 and 18,032 of the total variants observed. No nonsense or frameshift mutations were identified.

We focused our analysis of variants on those that were previously unreported and not inherited from the parental mouse strains, since these are the candidate variants that may alter the physiological state of the MutaMouse model compared to other mouse models. We constrained our analysis to variants that were present in genic regions from pathways that are relevant to genetic toxicology studies: genes involved in cancer progression, cell cycle, DNA repair, or xenobiotic metabolism (Table 2, Supplemental Table 2). When considering small variants (SNVs and indels) private to MutaMouse and identified as potentially deleterious, very few variants emerge: 26 for cancer genes, 32 for cell cycle genes, 5 for DNA repair genes, and 1 for xenobiotic metabolism. Even fewer of these variants were found across all the animals sequenced (Table 2). Overall, most of the identified variants already exist in other mouse strains. Furthermore, most variants do not affect coding sequences, and instead lie in regulatory regions (Fig. 3).

Within the genes of interest, we identified 22 genes that possess SNVs unique to MutaMouse that are also predicted to deleteriously affect the function of a gene product, as indicated by a SIFT score of $<0.05^{34}$ (SIFT was run as part of the VEP analysis; Table 3). Some of these genes (e.g., Rrs1, Hsp90ab1) possess multiple variants within their coding sequences, while others (e.g., Ahr) possess only one. Ahr is the only xenobiotic metabolizing gene that we identified containing a variant with a deleterious SIFT score (chr12:35,508,182 T $>$ C, which alters the codon Atc $>$ Gtc resulting in an I $>$ V amino acid change within exon 8). This variant was homozygous in every MutaMouse animal sequenced. We also found two DNA repair genes, Rev1 and $C d c 5 l$, with variants that result in amino acid substitution: chr1:38,088,013 T > C, chr1:38088020 C $>\mathrm{G}$ ( $\mathrm{K}>\mathrm{E}$ and $\mathrm{K}>\mathrm{N}$ amino acid changes) in Rev1; and chr17:45,407,947 G > A (causing a T $>M$ amino acid change) in Cdc5l. The remaining genes that possessed detrimental variants were involved with cell cycle regulation or cancer progression. We expected this result because these pathways contain 1,434 and 642 genes, respectively, which is more than DNA repair, with 408 genes, and xenobiotic metabolism, with 45 genes. Overall, although the SIFT algorithm predicted that some of these variants are deleterious, we have yet to discover phenotypic evidence that any of these pathways are significantly affected in MutaMouse. 


\section{A. SNPs and short indels}

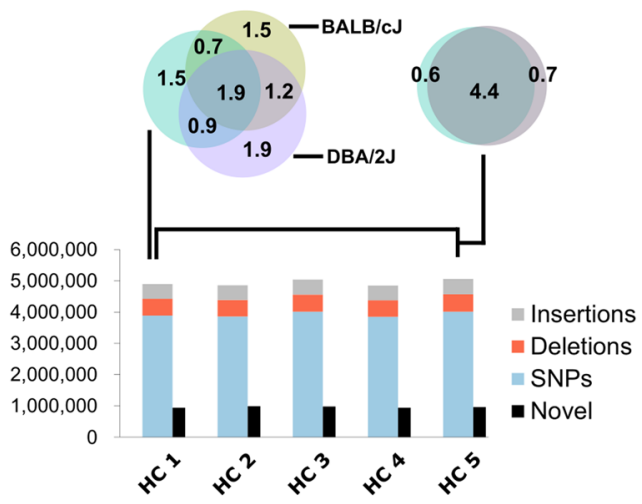

\section{Overlap in CNV Calls}

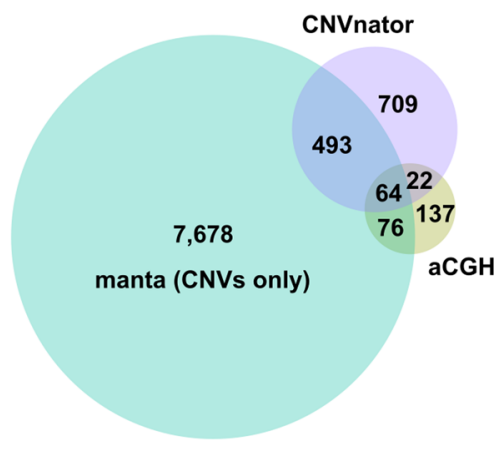

\section{B. Structural Variants}

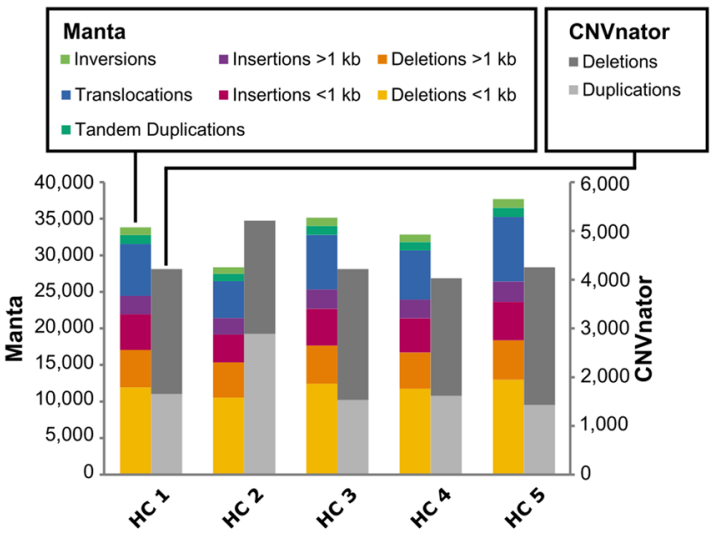

Figure 1. Summary of the number of genetic variants identified in MutaMouse animals from the Health Canada colony sequenced for this study. (A) The number of small variants discovered in each animal and the relative proportions of SNVs, insertions, and deletions. The number of novel variants (i.e., those not found in dbSNP v142) is shown as a black bar. A consistent number of small variants was observed between animals, with an average pairwise overlap of $85 \%$. A Venn diagram with the number of variants (in millions) shared by two representative MutaMouse individuals is shown above right, and a Venn diagram showing the overlap with parental strains DBA/2 and BALB/c is shown above left. (B) Structural variants (SVs) identified in MutaMouse using NGS data and two different algorithms (Manta and CNVnator). Nearly 10-fold more SVs were predicted by Manta, which uses paired-end information to determine discordant reads. CNVnator was used to predict the locations of copy number variants greater than $250 \mathrm{bp}$ in length. (C) The overlap in CNV calls between different methods. This analysis was limited to CNVs only found in all five animals. The majority of each call set does not overlap with the others, reinforcing the idea that multiple algorithms and methods should be used to call structural variants.

Of the 1,094 and 1,842 deletions that were found in all the mice using Manta and CNVnator (the NGS-based methods for SV calling), respectively, 13 deletions (Table 4) occurred in genes of toxicological interest. We predict that these deletions do not have a significant impact on protein function since they all fall in intergenic (upstream or downstream) or intronic regions. However, they may impact expression or transcript processing of the genes since regulatory regions and introns are deleted.

There is little overlap between NGS-based and aCGH CNV calls because the probe spacing for aCGH (on the order of kilobases) is much coarser than the resolution of NGS-based methods; thus, CNVnator is able to predict much smaller CNVs. Each of these methods is expected to provide complementary information to access different resolutions of SVs. To increase the reliability of our aCGH calls, we overlaid NGS coverage with each CNV call made using aCGH data. This revealed some instances where calls interpreted by the R-gada algorithm as one large event were actually composed of several small events (Fig. 4). Supplemental Table S3 describes the CNV calls made using aCGH and the CNV classification type (simple vs. complex) that we established using NGS coverage data to eliminate regions that R-gada predicted as CNVs. We classified events as complex SVs (encompassing multiple insertions and deletions) if they contained more than one CNV called by CNVnator. After this analysis, we classified 142 of the R-gada calls as simple and 156 as complex.

Transgene integration site. The transgene integration site is on the $\mathrm{B}$ region of chromosome $3^{35,36}$, but the precise breakpoints are unknown. We identified three long (PacBio) reads that overlapped the breakpoints, containing both mouse and transgene sequence. Surprisingly, the breakpoints were found at either end of a 


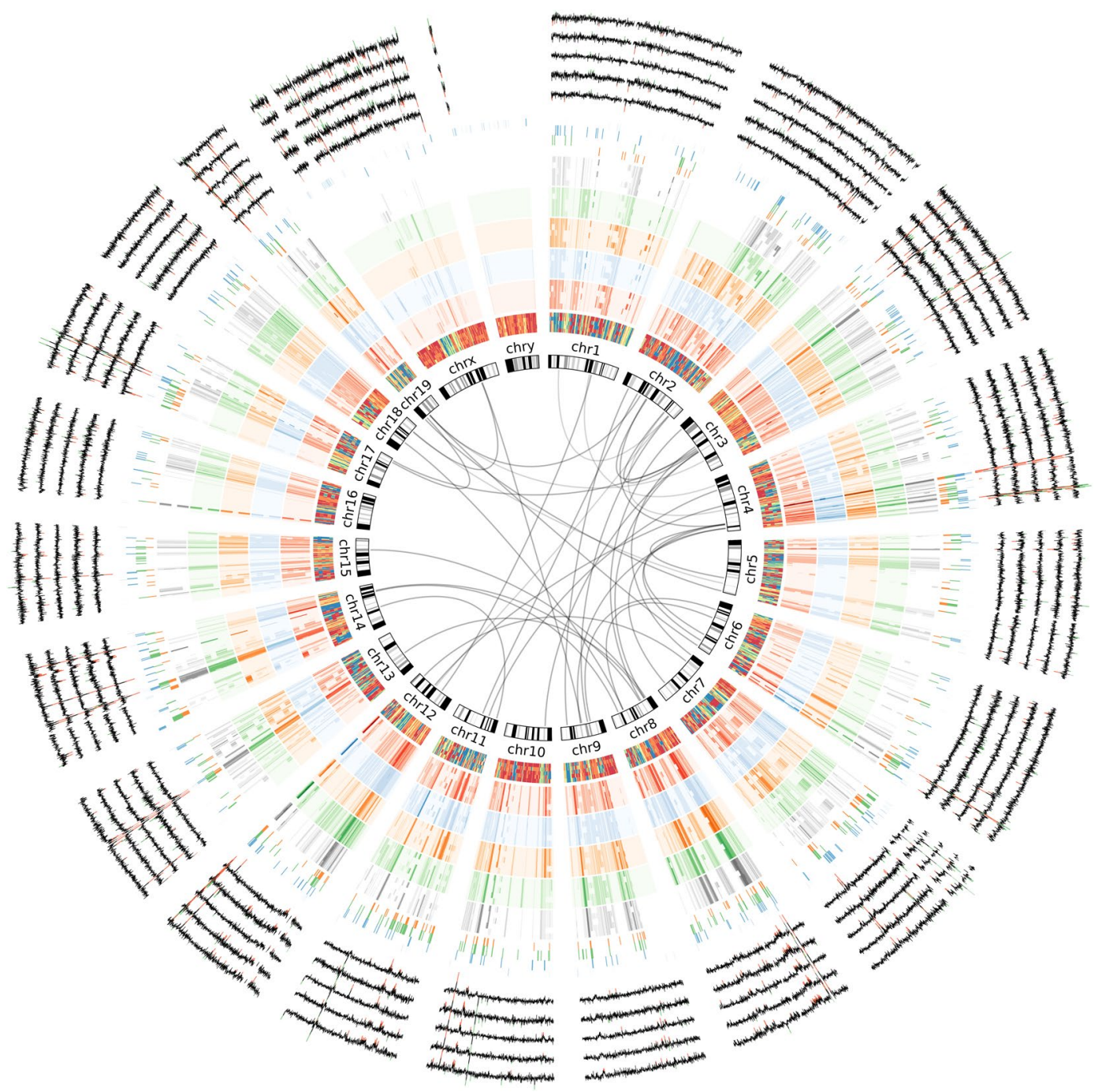

Figure 2. Circos $\operatorname{plot}^{54}$ showing genetic variation between five individual MutaMouse animals from a colony maintained at Health Canada. Links represent structural translocations identified in all mice sequenced. Each outside track has 5 individual components, one for each mouse sequenced (MutaMouse HC 1, 2, 3, 4, 5, from inside to out). The tracks from inside to outside represent (1) runs of homozygosity (where blue is homozygous and red is heterozygous - immediately adjacent to chromosome numbering), (2) Red track: SNV density per megabase of DNA sequence for all SNVs, (3) Blue track: SNV density for novel SNVs, (4) Orange track: SNV density for variants inherited from DBA/2J exclusively, (5) Green track: SNV density for variants inherited from $\mathrm{BALB} / \mathrm{cJ}$ exclusively, and (6) Grey track: SNV density for variants inherited from both parents. The next tracks show copy number variants for the parental strains $\mathrm{DBA} / 2 \mathrm{~J}$ and $\mathrm{BALB} / \mathrm{cJ}$ (orange and green, respectively), followed by copy number variants in MutaMouse predicted by CNVnator (blue). Finally, the line plots show the $\log 2$ values for aCGH probes averaged over 1 megabase intervals, with red highlights showing deleted segments and green highlights showing duplicated segments for each mouse.

465,426 bp homozygous deletion, accompanied by a 370 bp inversion detected by discordant mate pairs and identified by Manta (a deletion spanning chr3:37,971,249-38,436,675, with the inversion of sequence from chr3:38,436,306-38,436,675 joined to the left breakpoint; Fig. 5). In the mm10 reference genome, there is a long terminal repeat (RMER20A\#LTR/ERVK) present upstream of this locus. The deletion observed in MutaMouse is not present in either of the parental mouse strains, and therefore likely occurred concurrently with the integration of the transgene. We have observed this event in all MutaMouse animals analyzed to date, from both the HC and Covance colonies.

The complete sequence of the transgene in MutaMouse, as well as the precise breakpoints of insertion, were hitherto unknown. De novo assembly of NGS mate pair reads that were collected by first aligning them to a manually-assembled reference of $\lambda \mathrm{gt} 10^{35}$ revealed several constitutional SNVs and short indels that are found in every copy of the transgene in MutaMouse (Fig. 5; Table 5). These variants do not alter the protein coding sequences in the lac $Z$ gene region and the sequence of the transgene is otherwise identical to the manually assembled version. 


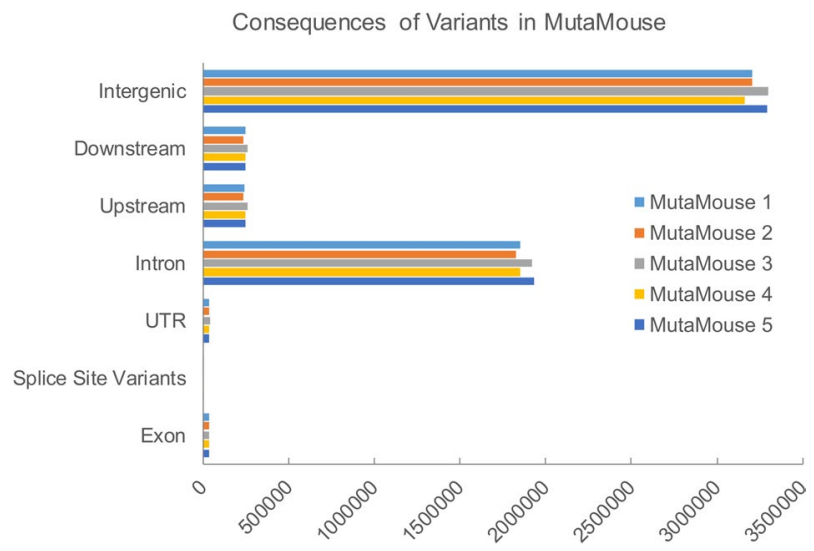

Figure 3. The number of predicted locations of variants sequenced in five MutaMouse animals. The majority of variants are found in intergenic regions, introns, or upstream and downstream non-coding sequences. Only $0.62 \%$ of variants (an average of 35,000 per animal) are found within exons.

Genetic similarity between two separate lineages of mutaMouse. To explore the genetic differences between the MutaMouse animals from the $\mathrm{HC}$ and Covance colonies, we compared the number of homozygous SNVs that were also found in the parental mice (Fig. 6A, left) or not (Fig. 6A, right), broken down further into which colony the SNV was present (both, HC only, or Covance only). The Covance colony retained marginally fewer parental SNVs than the HC colony $(942,713$ vs $1,046,884$ in total). The number of non-parental SNVs was comparable between the two colonies $(570,600$ and 574,935$)$. In total, when considering SNVs that were non-parental and homozygous, the HC and Covance colonies shared 181,821 SNVs, while 47,847 non-parental SNVs segregated in the HC colony and 17,677 segregated in the Covance colony. Furthermore, the two colonies segregate into separate branches on a phylogenetic tree based on their SNVs (Fig. 6B). Based on pairwise genotype concordance (Fig. 6C), mice originating from the two colonies are highly discordant (i.e., calculated as [\# of different sites]/[total sites]; discordance values range between 35.5 and 44.3 for animals between colonies). The two mice within the Covance colony are more similar to one another (discordance of 0.53), while mice from the HC colony show more discordance within their own group (discordance between 19.9 and 33.7).

To exclude that these results were due to differences in the median genome-wide read depth between the two colonies (54-81X for the HC colony, 28-32X for the Covance colony) we downsampled the sequenced data for the five $\mathrm{HC}$ mice to an average depth of 28-30X and found that there was no significant difference in the number of variants that were called (Supplemental Table S4). Similarly, there was no impact of downsampling the data from HC MutaMouse 2 generated with the same sequencing platform as the Covance mice (Supplemental Table S5).

\section{Discussion}

We generated the whole genome sequence of the MutaMouse model and characterized variants in multiple individuals from two geographically isolated colonies using a high sequence depth combined with aCGH. Analysis of these variants identified novel coding variants (mainly SNVs) that may influence genes involved in pathways related to toxicological responses, which is the primary use of this mouse model. No SVs that affected the coding sequence of these genes were identified, however, there were many SVs within the intronic, upstream, and downstream sequences that may modulate gene function. Characterization of the transgene integration site showed that it does not interrupt any genes. Finally, our work provides a reference sequence for future genomic studies on this important toxicological model.

A novel aspect of our investigation is the characterization of genetic variability between two MutaMouse colonies. Previous work has focused on the genetic variants found in different strains of inbred mice ${ }^{25,30,33,37-39}$. Although not inbred, HC has maintained a MutaMouse colony from a small founding population over 78 generations since 1990. Despite this, we found that there is a high level of genetic diversity present within the HC colony. In fact, the numbers of private (unshared) variants between animals sequenced from this colony ranged from 54,000 to 148,000 (Fig. 1A). Overall, our results suggest that the MutaMouse colony at HC is genetically heterogeneous and shows that new genetic variants have arisen after 78 generations. Conversely, the Covance colony had fewer private variants $(24,259$ to 27,277$)$. We excluded that these results were due to the difference in coverage obtained from these two colonies (Tables 1 and S4) or to library type (Supplemental Table S5). Thus, we conclude that the reduce genetic diversity in the Covance colony is partly due to a population bottleneck experienced in the Covance colony in 2006 when the colony was restarted using a few pairs of animals.

Littermates from one inbred mouse colony reportedly vary from each other by approximately 985 SNVs genome wide ${ }^{40}$. We observed 50-150 fold more private SNVs between littermates. A possible explanation is that outbred colonies possess higher number of private variants when compared to inbred populations, with the important caveat that cross-study comparisons may be unreliable (e.g., different reference genomes were used, different variant calling workflows were followed). Overall, the selection of mouse strains used in an experiment requires considering the trade-offs between reducing variability in the results of toxicological assays by using 


\begin{tabular}{|c|c|c|c|c|c|c|c|c|c|c|c|c|}
\hline \multirow[b]{2}{*}{ Animals } & \multicolumn{3}{|c|}{ Cancer Progression ${ }^{\mathrm{a}}$ (642 genes) } & \multicolumn{3}{|c|}{ Cell Cycle ${ }^{b}(1,434$ genes $)$} & \multicolumn{3}{|c|}{ DNA repair ${ }^{b}$ (408 genes) } & \multicolumn{3}{|c|}{ Xenobiotic Metabolismb (45 genes) } \\
\hline & $\begin{array}{l}\text { Variants } \\
\text { within } \\
\text { genes }\end{array}$ & $\begin{array}{l}\text { Non } \\
\text { synonymous }\end{array}$ & $\begin{array}{l}\text { SIFT score } \\
\text { deleterious }^{\mathrm{c}}\end{array}$ & $\begin{array}{l}\text { Variants } \\
\text { within } \\
\text { genes }\end{array}$ & $\begin{array}{l}\text { Non } \\
\text { synonymous }\end{array}$ & $\begin{array}{l}\text { SIFT score } \\
\text { deleterious }^{\mathrm{d}}\end{array}$ & $\begin{array}{l}\text { Variants } \\
\text { within } \\
\text { genes }\end{array}$ & $\begin{array}{l}\text { Non } \\
\text { synonymous }\end{array}$ & $\begin{array}{l}\text { SIFT score } \\
\text { deleterious }^{\mathrm{c}}\end{array}$ & \begin{tabular}{|l|} 
Variants \\
within \\
genes
\end{tabular} & $\begin{array}{l}\text { Non } \\
\text { synonymous }\end{array}$ & $\begin{array}{l}\text { SIFT score } \\
\text { deleterious }^{c}\end{array}$ \\
\hline $\begin{array}{l}\text { MutaMouse } \\
1\end{array}$ & $\begin{array}{l}93,708 \\
(15,099)^{c}\end{array}$ & $\begin{array}{l}301 \\
(34)\end{array}$ & $\begin{array}{l}52 \\
(8)\end{array}$ & $\begin{array}{l}133,383 \\
(21,452)\end{array}$ & $\begin{array}{l}659 \\
(47)\end{array}$ & $\begin{array}{l}98 \\
(23)\end{array}$ & $\begin{array}{l}35,592 \\
(5,567)\end{array}$ & $\begin{array}{l}244 \\
(3)\end{array}$ & $\begin{array}{l}27 \\
(0)\end{array}$ & $\begin{array}{l}3,483 \\
(630)\end{array}$ & $\begin{array}{l}13 \\
(1)\end{array}$ & $\begin{array}{l}2 \\
(1)\end{array}$ \\
\hline $\begin{array}{l}\text { MutaMouse } \\
2\end{array}$ & $\begin{array}{l}80,922 \\
(14,455)\end{array}$ & $\begin{array}{l}313 \\
(38)\end{array}$ & $\begin{array}{l}54 \\
(10)\end{array}$ & $\begin{array}{l}135,318 \\
(21,943)\end{array}$ & $\begin{array}{l}598 \\
(47)\end{array}$ & $\begin{array}{l}96 \\
(24)\end{array}$ & $\begin{array}{l}34,416 \\
(5,475)\end{array}$ & $\begin{array}{l}222 \\
(9)\end{array}$ & $\begin{array}{l}27 \\
(4)\end{array}$ & $\begin{array}{l}3,295 \\
(586)\end{array}$ & $\begin{array}{l}11 \\
(1)\end{array}$ & $\begin{array}{l}2 \\
(1)\end{array}$ \\
\hline $\begin{array}{l}\text { MutaMouse } \\
3\end{array}$ & $\begin{array}{l}98,330 \\
(15,681)\end{array}$ & $\begin{array}{l}354 \\
(45)\end{array}$ & $\begin{array}{l}67 \\
(20) \\
\end{array}$ & $\begin{array}{l}147,108 \\
(23,716)\end{array}$ & $\begin{array}{l}745 \\
(58) \\
\end{array}$ & $\begin{array}{l}117 \\
(29) \\
\end{array}$ & $\begin{array}{l}39,493 \\
(6,055)\end{array}$ & $\begin{array}{l}246 \\
(3)\end{array}$ & $\begin{array}{l}27 \\
(1)\end{array}$ & $\begin{array}{l}3,469 \\
(643)\end{array}$ & $\begin{array}{l}11 \\
(1)\end{array}$ & $\begin{array}{l}2 \\
(1)\end{array}$ \\
\hline $\begin{array}{l}\text { MutaMouse } \\
4\end{array}$ & $\begin{array}{l}89,172 \\
(14,906) \\
\end{array}$ & $\begin{array}{l}324 \\
(44) \\
\end{array}$ & $\begin{array}{l}65 \\
(20) \\
\end{array}$ & \begin{tabular}{|l|}
136,668 \\
$(21,821)$ \\
\end{tabular} & $\begin{array}{l}621 \\
(51) \\
\end{array}$ & $\begin{array}{l}102 \\
(26)\end{array}$ & \begin{tabular}{|l|}
37,618 \\
$(5,802)$ \\
\end{tabular} & $\begin{array}{l}238 \\
(7) \\
\end{array}$ & $\begin{array}{l}30 \\
(3) \\
\end{array}$ & $\begin{array}{l}3,326 \\
(583) \\
\end{array}$ & $\begin{array}{l}11 \\
(1) \\
\end{array}$ & \begin{tabular}{|l|}
2 \\
$(1)$
\end{tabular} \\
\hline $\begin{array}{l}\text { MutaMouse } \\
5\end{array}$ & $\begin{array}{l}110,565 \\
(16,748) \\
\end{array}$ & $\begin{array}{l}327 \\
(39)\end{array}$ & $\begin{array}{l}56 \\
(11)\end{array}$ & $\begin{array}{l}138,297 \\
(22,785) \\
\end{array}$ & \begin{tabular}{|l|}
645 \\
$(49)$
\end{tabular} & $\begin{array}{l}98 \\
(23) \\
\end{array}$ & $\begin{array}{l}39,869 \\
(6,156)\end{array}$ & $\begin{array}{l}258 \\
(5) \\
\end{array}$ & $\begin{array}{l}28 \\
(1)\end{array}$ & $\begin{array}{l}3,278 \\
(592) \\
\end{array}$ & $\begin{array}{l}11 \\
(1) \\
\end{array}$ & \begin{tabular}{|l}
2 \\
$(1)$
\end{tabular} \\
\hline $\begin{array}{l}\text { MutaMouse } \\
\text { Covance } \\
\text { Female }\end{array}$ & $\begin{array}{l}71,057 \\
(9,512)\end{array}$ & $\begin{array}{l}2,379 \\
(37)\end{array}$ & $\begin{array}{l}42 \\
(20)\end{array}$ & $\begin{array}{l}111,611 \\
(14,304)\end{array}$ & $\begin{array}{l}541 \\
(31)\end{array}$ & $\begin{array}{l}91 \\
(17)\end{array}$ & $\begin{array}{l}28,344 \\
(3,663)\end{array}$ & $\begin{array}{l}198 \\
(6)\end{array}$ & $\begin{array}{l}29 \\
(3)\end{array}$ & $\begin{array}{l}2,932 \\
(362)\end{array}$ & $\begin{array}{l}10 \\
(1)\end{array}$ & $\begin{array}{l}2 \\
(1)\end{array}$ \\
\hline $\begin{array}{l}\text { MutaMouse } \\
\text { Covance } \\
\text { Male }\end{array}$ & $\begin{array}{l}71,513 \\
(9,820)\end{array}$ & $\begin{array}{l}242 \\
(44)\end{array}$ & $\begin{array}{l}43 \\
(22)\end{array}$ & $\begin{array}{l}112,389 \\
(14,734)\end{array}$ & $\begin{array}{l}545 \\
(36)\end{array}$ & $\begin{array}{l}90 \\
(17)\end{array}$ & $\begin{array}{l}28,474 \\
(3,478)\end{array}$ & $\begin{array}{l}199 \\
(7)\end{array}$ & $\begin{array}{l}29 \\
(3)\end{array}$ & $\begin{array}{l}2,934 \\
(354)\end{array}$ & $\begin{array}{l}10 \\
(1)\end{array}$ & $\begin{array}{l}2 \\
(1)\end{array}$ \\
\hline Common & \begin{tabular}{|l|}
55,389 \\
$(7,682)$
\end{tabular} & $\begin{array}{l}219 \\
(22)\end{array}$ & $\begin{array}{l}36 \\
(5)\end{array}$ & \begin{tabular}{|l|}
95,155 \\
$(12,129)$
\end{tabular} & \begin{tabular}{|l|}
479 \\
$(35)$
\end{tabular} & $\begin{array}{l}68 \\
(18)\end{array}$ & $\begin{array}{l}27,121 \\
(3,309)\end{array}$ & $\begin{array}{l}184 \\
(2)\end{array}$ & $\begin{array}{l}19 \\
(0)\end{array}$ & $\begin{array}{l}2,979 \\
(358)\end{array}$ & $\begin{array}{l}11 \\
(1)\end{array}$ & $\begin{array}{l}2 \\
(1)\end{array}$ \\
\hline Total & $\begin{array}{l}132,221 \\
(24,470)\end{array}$ & $\begin{array}{l}407 \\
(66)\end{array}$ & $\begin{array}{l}71 \\
(26)\end{array}$ & $\begin{array}{l}180,472 \\
(34,966)\end{array}$ & \begin{tabular}{|l|}
870 \\
$(74)$
\end{tabular} & $\begin{array}{l}129 \\
(32)\end{array}$ & \begin{tabular}{|l|}
46,554 \\
$(8,808)$
\end{tabular} & $\begin{array}{l}280 \\
(11)\end{array}$ & $\begin{array}{l}34 \\
(5)\end{array}$ & $\begin{array}{l}3,938 \\
(904)\end{array}$ & $\begin{array}{l}13 \\
(1)\end{array}$ & \begin{tabular}{|l|}
2 \\
$(1)$
\end{tabular} \\
\hline
\end{tabular}

Table 2. Variants (SNVs and short indels) identified in MutaMouse in the genes from pathways that are important for genetic toxicology studies. ${ }^{\mathrm{a} C O S M I C}$ cancer genes (Mouse homologs). ${ }^{\mathrm{b}}$ Retrieved using BioMart. 'SIFT scores calculated using Ensembl Variant Effect Predictor; only canonical transcripts reported (i.e., only one consequence is reported per variant). ${ }^{\mathrm{d}}$ Not reported in dbSNP $142 .{ }^{\text {eSubset }}$ of variants that are present in all

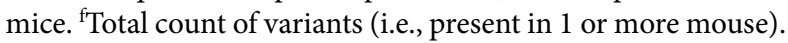

inbred lines, or capturing more biological diversity to more closely reflect different responses that may exist in human populations.

Since the MutaMouse model is primarily used in genetic toxicology studies, we focused our in silico analysis on variants that are private to MutaMouse and carry potential phenotypic effects for genes involved in DNA repair, xenobiotic metabolism, cancer progression, and cell cycle regulation. For example, we identified a SNV within the $A h r$ gene that was present in all sequenced animals and that was predicted to affect the function of the protein. This is of special interest to research groups that regularly use aromatic hydrocarbons as model mutagens, since it may influence gene expression in response to certain chemical insults. Moreover, chemicals and drugs that are AhR agonists play important roles in carcinogenesis ${ }^{41}$. However, previous work indicates that the $A h r$ gene in MutaMouse has a normal phenotype because: (a) Cyp1A1, a downstream target of AhR, is highly inducible following exposure to PAHs and (b) these mice effectively metabolize aromatic compounds (a process that is regulated by $\mathrm{AhR}$ ) resulting in very strong induction of mutations $\mathrm{s}^{8,42}$. Additionally, there were no deleterious variants in genes involved in xenobiotic metabolism processes (e.g., cytochrome P450s). This serves as an example that in silico predictions, such as the biological effect of a SNV or mutation, always need phenotypic anchoring ${ }^{43}$. There were no potentially deleterious mutations in P53 and its key downstream targets, supporting the observation that the MutaMouse possesses an intact P53 response ${ }^{44,45}$. Overall, our results raise no concern about the utility of MutaMouse as a model for genetic toxicology studies.

Some mouse genomic studies have historically been limited because they used analytical methods that did not call SVs, which make up a large base-pair number component of variation between individuals ${ }^{46}$. The putative SVs identified in MutaMouse are far less numerous than the SNVs and short indels discovered using the HaplotypeCaller (Fig. 1B). We used multiple algorithms (CNVnator and Manta) to call distinct types of SVs. $\mathrm{CNVnator}{ }^{47}$ uses a depth-based approach in which coverage is binned over a range (250 bp regions in this study) and adjacent bins are compared to identify areas of increased coverage (duplications) or decreased coverage (deletions). Manta ${ }^{48}$ uses split read, read pair, and read depth information to discover potential rearrangements (translocations) as well as tandem duplications, inversions, deletions, and insertions. In addition to NGS data and the associated algorithms, we also used aCGH to call CNVs (large insertions or deletions, typically on the scale $>1 \mathrm{~kb}$, depending on probe distances). We identified $\sim 35,000$ SVs per genome using Manta, $\sim 4,000 \mathrm{CNVs}$ using CNVnator, and $\sim 300 \mathrm{CNV}$ s using aCGH. We show these results in Fig. 2, which also shows runs of homozygosity and SNV density at a genome-wide scale for all the animals sequenced. Importantly though, we found no SVs that altered the coding sequence of toxicologically relevant genes (Table 4).

NGS and aCGH provide highly complementary data types and can be used to strengthen one another. We found that analyzing NGS coverage data together with aCGH data added precision to CNV calls generated by aCGH. We found that aCGH is highly sensitive and can accurately predict the locations of CNVs (both deletions and gains; Fig. 4A,B). However, the non-uniform spacing of array probes obscured the true nature of a subset of these events (Fig. 4C,D). Specifically, erroneous calls of single large CNVs were made based on the aCGH data, which the NGS data revealed to be composed of a complex series of small insertions and/or deletions. This is important for adding clarity to datasets; specifically, this resolves the apparently contradictory result that 


\begin{tabular}{|c|c|c|c|c|c|c|c|c|c|}
\hline \multirow{2}{*}{$\begin{array}{l}\text { Gene } \\
\text { Symbol }\end{array}$} & \multicolumn{5}{|c|}{$\begin{array}{l}\text { Number of SNVs in gene for each } \\
\text { animal }\end{array}$} & \multirow{2}{*}{$\begin{array}{l}\text { Chromosomal position(s) and } \\
\text { nucleotide change(s) }\end{array}$} & \multirow{2}{*}{$\begin{array}{l}\text { Amino acid change } \\
\text { (codon change) }\end{array}$} & \multirow[b]{2}{*}{ Protein $^{\mathrm{a}}$} & \multirow[b]{2}{*}{ Functions } \\
\hline & HC 1 & HC 2 & HC 3 & HC 4 & HC 5 & & & & \\
\hline Ahr & 1 & 1 & 1 & 1 & 1 & chr12:35508182 T $>C$ & I/V (Atc/Gtc) & Aryl hydrocarbon receptor & $\begin{array}{l}\text { Xenobiotic } \\
\text { metabolism, Cell } \\
\text { cycle }\end{array}$ \\
\hline Arpp19 & 1 & 1 & 1 & 1 & 1 & chr9:75056711C $>$ G & $\mathrm{P} / \mathrm{R}(\mathrm{cCg} / \mathrm{cGg})$ & cAMP-regulated phosphoprotein 19 & Cell cycle \\
\hline $\mathrm{Cbl}$ & 0 & 0 & 0 & 1 & 2 & $\begin{array}{l}\text { chr9:44151504C }>\mathrm{T} \\
\text { chr9:44151527 }>\mathrm{T}\end{array}$ & $\begin{array}{l}\mathrm{A} / \mathrm{T}(\mathrm{Gct} / \mathrm{Act}) \\
\mathrm{I} / \mathrm{N}(\mathrm{aTt} / \mathrm{aAt})\end{array}$ & E3 ubiquitin-protein ligase CBL & Cancer progression \\
\hline Ccnb2 & 0 & 0 & 0 & 1 & 0 & chr9:70410215G $>$ A & $\mathrm{T} / \mathrm{M}(\mathrm{aCg} / \mathrm{aTg})$ & G2/mitotic-specific cyclin-B2 & Cell cycle \\
\hline Ccnb3 & 1 & 1 & 1 & 1 & 1 & chrX:7025679T >A & $\mathrm{N} / \mathrm{I}(\mathrm{aAc} / \mathrm{aTc})$ & G2/mitotic-specific cyclin-B3 & Cell cycle \\
\hline Cdc5l & 0 & 0 & 1 & 0 & 0 & chr17:45407947 G > A, & $\mathrm{T} / \mathrm{M}(\mathrm{aCg} / \mathrm{aTg})$ & Cell division cycle 5-like protein & $\begin{array}{l}\text { DNA repair, Cell } \\
\text { cycle }\end{array}$ \\
\hline Cdk4 & 0 & 0 & 1 & 0 & 1 & $\operatorname{chr} 10: 127064302 \mathrm{G}>\mathrm{A}$ & $\mathrm{A} / \mathrm{T}(\mathrm{Gcc} / \mathrm{Acc})$ & Cyclin-dependent kinase 4 & $\begin{array}{l}\text { Cancer progression, } \\
\text { Cell cycle }\end{array}$ \\
\hline Cenpt & 0 & 0 & 0 & 1 & 0 & chr8:105845366 G > A & $\mathrm{R} / \mathrm{C}(\mathrm{Cgc} / \mathrm{Tgc})$ & Centromere protein $\mathrm{T}$ & Cell cycle \\
\hline Eif4a2 & 0 & 0 & 2 & 2 & 0 & 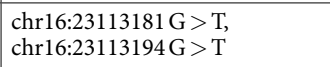 & $\begin{array}{l}\text { G/V (gGt/gTt), } \\
\mathrm{R} / \mathrm{S} \text { (agG/agT) }\end{array}$ & Eukaryotic initiation factor 4A-II & Cancer progression \\
\hline Hsp90ab1 & 0 & 1 & 9 & 9 & 1 & 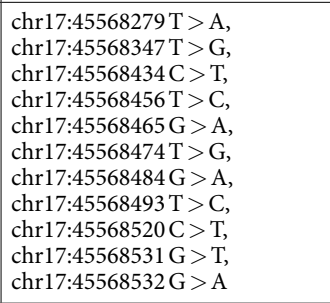 & $\begin{array}{l}\text { D/V (gAt/gTt), } \\
\text { K/N (aaA/aaC), } \\
\text { M/I (atG/atA), } \\
\text { D/G (gAc/gGc), } \\
\text { A/V (gCa/gTa), } \\
\text { K/T (aAg/aCg), } \\
\text { R/W (Cgg/Tgg), } \\
\text { N/D (Aac/Gac), } \\
\text { V/M (Gtg/Atg), } \\
\text { P/H (cCc/cAc), } \\
\text { P/S (Ccc/Tcc) }\end{array}$ & Heat shock protein HSP 90-beta & Cancer progression \\
\hline Mapk6 & 1 & 1 & 1 & 1 & 1 & chr9:75388680 T >A & $\mathrm{Q} / \mathrm{L}(\mathrm{cAg} / \mathrm{cTg})$ & Mitogen-activated protein kinase 6 & Cell cycle \\
\hline Mplkip & 2 & 2 & 2 & 2 & 2 & $\begin{array}{l}\text { chr13:17695605C > T, } \\
\text { chr13:17695681C }>A\end{array}$ & $\begin{array}{l}\mathrm{R} / \mathrm{W}(\mathrm{Cgg} / \mathrm{Tgg}) \\
\mathrm{P} / \mathrm{Q}(\mathrm{cCg} / \mathrm{cAg})\end{array}$ & $\begin{array}{l}\text { M-phase-specific PLK1-interacting } \\
\text { protein }\end{array}$ & Cell cycle \\
\hline Msi2 & 1 & 1 & 1 & 1 & 1 & $\operatorname{chr} 11: 88687461 \mathrm{C}>\mathrm{A}$ & $\mathrm{V} / \mathrm{F}(\mathrm{Gtc} / \mathrm{Ttc})$ & $\begin{array}{l}\text { RNA-binding protein Musashi } \\
\text { homolog }\end{array}$ & Cancer progression \\
\hline Nabp2 & 0 & 1 & 0 & 0 & 1 & chr10:128408557 C > T & $\mathrm{G} / \mathrm{S}(\mathrm{Ggc} / \mathrm{Agc})$ & SOSS complex subunit B1 & $\begin{array}{l}\text { DNA repair, Cell } \\
\text { cycle }\end{array}$ \\
\hline Nanog & 1 & 0 & 1 & 1 & 0 & chr6:122707814T > G & $\mathrm{L} / \mathrm{W}(\mathrm{tTg} / \mathrm{tGg})$ & Homeobox protein & Cell cycle \\
\hline Nek1 & 1 & 1 & 1 & 1 & 1 & chr8:61054575C > A & $\mathrm{R} / \mathrm{S}$ (Cgt/Agt) & Serine/threonine-protein kinase & Cell cycle \\
\hline Rev1 & 0 & 2 & 0 & 2 & 0 & $\begin{array}{l}\text { chrl:38088013 T >C, } \\
\text { chrl:38088020 C >G }\end{array}$ & $\begin{array}{l}\text { K/E (Aaa/Gaa), } \\
\text { K/N (aaG/aaC) }\end{array}$ & DNA repair protein REV1 & DNA repair \\
\hline Rprd1b & 0 & 1 & 0 & 0 & 0 & chr2:158047932G $>\mathrm{T}$ & $\mathrm{K} / \mathrm{N}(\mathrm{aaG} / \mathrm{aaT})$ & $\begin{array}{l}\text { Regulation of nuclear pre-mRNA } \\
\text { domain-containing protein } 1 \mathrm{~B}\end{array}$ & Cell cycle \\
\hline Rrs1 & 13 & 12 & 13 & 13 & 13 & 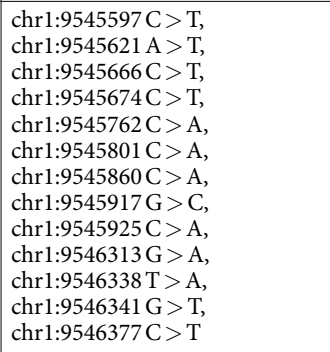 & $\begin{array}{l}\text { T/M (aCg/aTg), } \\
\text { E/V (gAg/gTg), } \\
\text { T/I (aCc/aTc), } \\
\text { R/C (Cgc/Tgc), } \\
\text { P/Q (cCg/cAg), } \\
\mathrm{P} / \mathrm{Q}(\mathrm{cCg} / \mathrm{cAg}), \\
\mathrm{R} / \mathrm{S}(\mathrm{Cgc/Agc}), \\
\text { V/L (Gtg/Ctg), } \\
\mathrm{D} / \mathrm{E}(\mathrm{gaC} / \mathrm{gaA}), \\
\text { E/K (Gag/Aag), } \\
\mathrm{L} / \mathrm{H}(\mathrm{cTt} / \mathrm{cAt}), \\
\mathrm{R} / \mathrm{L}(\mathrm{cGa} / \mathrm{cTa}), \\
\text { T/M (aCg/aTg) }\end{array}$ & $\begin{array}{l}\text { Ribosome biogenesis regulatory } \\
\text { protein homolog }\end{array}$ & Cell cycle \\
\hline Tdpoz2 & 1 & 1 & 1 & 1 & 1 & chr3:93652273G $>C$ & $\mathrm{~L} / \mathrm{V}(\mathrm{Ctc} / \mathrm{Gtc})$ & $\begin{array}{l}\text { TD and POZ domain-containing } \\
\text { protein } 2\end{array}$ & Cancer progression \\
\hline Thrap3 & 3 & 1 & 3 & 3 & 3 & $\begin{array}{l}\text { chr4:126165462 T >C, } \\
\text { chr4:126165536G }>A, \\
\text { chr4:126165542 }>\text { A }\end{array}$ & $\begin{array}{l}\mathrm{I} / \mathrm{M}(\mathrm{atA} / \mathrm{atG}) \\
\mathrm{R} / \mathrm{W}(\mathrm{Cgg} / \mathrm{Tgg}), \\
\mathrm{R} / \mathrm{W}(\mathrm{Cgg} / \mathrm{Tgg})\end{array}$ & $\begin{array}{l}\text { Thyroid hormone receptor- } \\
\text { associated protein } 3\end{array}$ & Cancer progression \\
\hline Tjp3 & 0 & 0 & 1 & 0 & 0 & chr10:81274478 T $>$ G & $(\mathrm{Atc} / \mathrm{Ctc})$ & Tight junction protein $\mathrm{ZO}-3$ & Cell cycle \\
\hline Total & 29 & 32 & 46 & 45 & 32 & & & & \\
\hline
\end{tabular}

Table 3. Genes in MutaMouse affected by potentially deleterious single nucleotide variants (as determined by

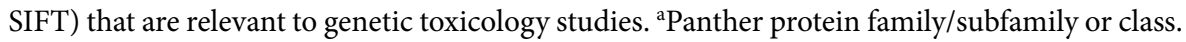

CNV calls from aCGH overlapped with SNV calls. Instead, these types of CNVs should be considered complex genomic regions where multiple chromosomal breaks occurred in a single span, along with potential mutations, similar to chromothripsis or other types of complex genomic rearrangements ${ }^{49}$. As NGS is becoming more affordable and the algorithms used for variant calling improve, both methodologies should be used to more precisely characterize SVs. 


\begin{tabular}{|c|c|c|c|c|c|c|}
\hline Gene & $\begin{array}{l}\text { Predicted effects of } \\
\text { variant }\end{array}$ & Chromosomal coordinates ${ }^{\mathrm{a}}$ & $\begin{array}{l}\text { Number of } \\
\text { deleted bases }\end{array}$ & Protein & Functions & $\begin{array}{l}\text { Copy numbers } \\
\text { observed in } \\
\text { MutaMouse } \\
\text { population }\end{array}$ \\
\hline Grk5 & intron variant & chr19:60925302-60934033 & 8,731 & G protein-coupled receptor kinase 5 & Cell cycle & 0 or 1 \\
\hline Kif5b & downstream gene variant & chr18:6196144-6199729 & 3,585 & Kinesin-1 heavy chain & Cancer progression & 0 or 1 \\
\hline Cdh11 & intron variant & chr8:102660453-102663326 & 2,873 & Cadherin-11 & Cancer progression & 0 \\
\hline Tnks & intron variant & chr8:34882786-34885003 & 2,217 & Tankyrase-1 & Cell cycle & 0 \\
\hline Dctn6 & downstream gene variant & chr8:34087155-34089063 & 1,908 & Dynactin subunit 6 & Cell cycle & 0 \\
\hline Nrg1 & intron variant & chr8:31969110-31970943 & 1,833 & Neuregulin 1 & Cancer progression & 0 \\
\hline Ncoa2 & upstream gene variant & chr1:13378446-13380234 & 1,788 & Nuclear receptor coactivator 2 & Cancer progression & 0 \\
\hline Arhgap26 & $\begin{array}{l}\text { intron variant, non- } \\
\text { coding transcript variant }\end{array}$ & chr18:38663457-38665186 & 1,729 & Rho GTPase-activating protein 26 & Cancer progression & 0 or 1 \\
\hline $\operatorname{Rec} 114$ & intron variant & chr9:58685759-58687011 & 1,252 & Meiotic recombination protein REC114 & Cell cycle & 0 or 1 \\
\hline Wdr62 & intron variant & chr7:30275960-30276784 & 824 & WD repeat-containing protein 62 & Cell cycle & 0 \\
\hline Prkdc & intron variant & chr16:15638729-15639323 & 594 & DNA-dependent protein kinase catalytic subunit & $\begin{array}{l}\text { Cell cycle, DNA } \\
\text { repair }\end{array}$ & 0 \\
\hline $\mathrm{Kmt2e}$ & intron variant & chr5:23494281-23494730 & 449 & Histone-lysine N-methyltransferase $2 \mathrm{E}$ & Cell cycle & 0 \\
\hline Fancc & intron variant & chr13:63480021-63480458 & 437 & Fanconi anemia group $\mathrm{C}$ protein homolog & $\begin{array}{l}\text { Cancer progression, } \\
\text { DNA repair }\end{array}$ & 0 \\
\hline
\end{tabular}

Table 4. Genes in MutaMouse affected by potentially detrimental deletions discovered with both CNVnator and Manta (NGS-based tools) that are relevant to genetic toxicology studies. ${ }^{\mathrm{a} C o o r d i n a t e s ~ r e p o r t e d ~ b y ~ M a n t a ~}$ are shown since they allow nucleotide resolution, while CNVnator reports only $250 \mathrm{bp}$ bin locations.

Our results are in line with a recent study ${ }^{28}$ that used a combination of short-read and long-read technology with the goal of improving the reference assembly of C57BL/6J ("Eve"). They were able to close previously open gaps in the assembly, resolve repeats, detect novel isoforms, and characterize structural variants with their approach. Their conclusion that genetic drift resulting from bottlenecks occurs frequently in mouse populations reflects precisely what we observed in our study. This support the concept of using different sequencing technologies in tandem to better characterize the genetics of mouse strains currently in use.

It is known that the MutaMouse $\lambda$ gt10-lac $Z$ reporter transgene is found at a single chromosomal location on chromosome 3 (B region) ${ }^{36}$. Later qPCR work detailed that the transgene is present in $\sim 29$ head to tail copies ${ }^{35}$. The authors also identified several in vivo rearrangements of the transgene, although the precise breakpoints of integration, and whether the integration interrupted any genes, were not determined. We found that integration of the $\lambda$ gt10 transgene occurred in a complex manner and was accompanied by a large deletion of nearly 0.5 Mbp of genomic DNA from MutaMouse. The nearby long terminal repeat retrotransposon (RMER20A\#LTR/ ERVK) may have played a role in the integration event and was likely the reason that the genomic coordinates of the transgene were obscured when screening using short-read technology. Long-read PacBio sequence data were key to resolving the integration region (Fig. 4), since the Illumina reads adjacent to and overlapping the insertion breakpoints were too short to extend into the lambda phage shuttle vector.

Characterized of the breakpoints and integration site demonstrated that no critical genes were interrupted or deleted by transgene integration (although the lincRNA 5430434I15Rik was deleted and predicted gene Gm20755 was truncated). At the downstream breakpoint, there was a 2 bp microhomology that is consistent with the microhomology-mediated break-induced replication mechanism ${ }^{50}$. However, the upstream breakpoint consists of a $370 \mathrm{bp}$ inversion in which mouse genomic sequence that originates from an area past the downstream breakpoint was placed before the insertion of four apparently random nucleotides, followed by the insertion of a transgene monomer. This is may have occurred through the hijacking of a repair process such as non-homologous end joining (NHEJ) by the foreign $\lambda$ gt $10 \mathrm{DNA}$, although it is unclear whether the $370 \mathrm{bp}$ inversion was part of an independent event. At both breakpoints, the transgene monomer is missing one of the cohesive ends, and therefore cannot be packaged into phage particles. The nonfunctional transgene monomer at the upstream breakpoint does not contain the replication origin. Our results provide some important mechanistic insight into how these random genomic integration events occur. It is becoming more apparent that structural genomic rearrangements are not uncommon in mammalian genomes and can occur via a wide range of mechanisms ${ }^{51}$. Our results support the hypothesis that complex structural rearrangement events require little homology to form and thus, they can form anywhere along the DNA.

Our study provides a detailed genetic analysis of the commonly used MutaMouse model that confirms its efficacy for genetic toxicology research and enhances the ability to apply new analytical options for future researchers. By sequencing multiple closely related individuals, we explored the variability in closely related mice within an outbred colony to show that a high level of diversity is present. Though we found several potentially functional SNVs, they do not appear to alter the expected functions, highlighting that such results always require phenotypic anchoring. In addition, we found that SVs did not affect coding sequences in genes for pathways relevant to genetic toxicology testing. We discovered that the genetic alteration accompanying transgene integration was a highly complex event, although it did not modify any genes. The MutaMouse genome contributes data for understanding quantitative trait loci and provide important genetic context to studies using this model. 
A
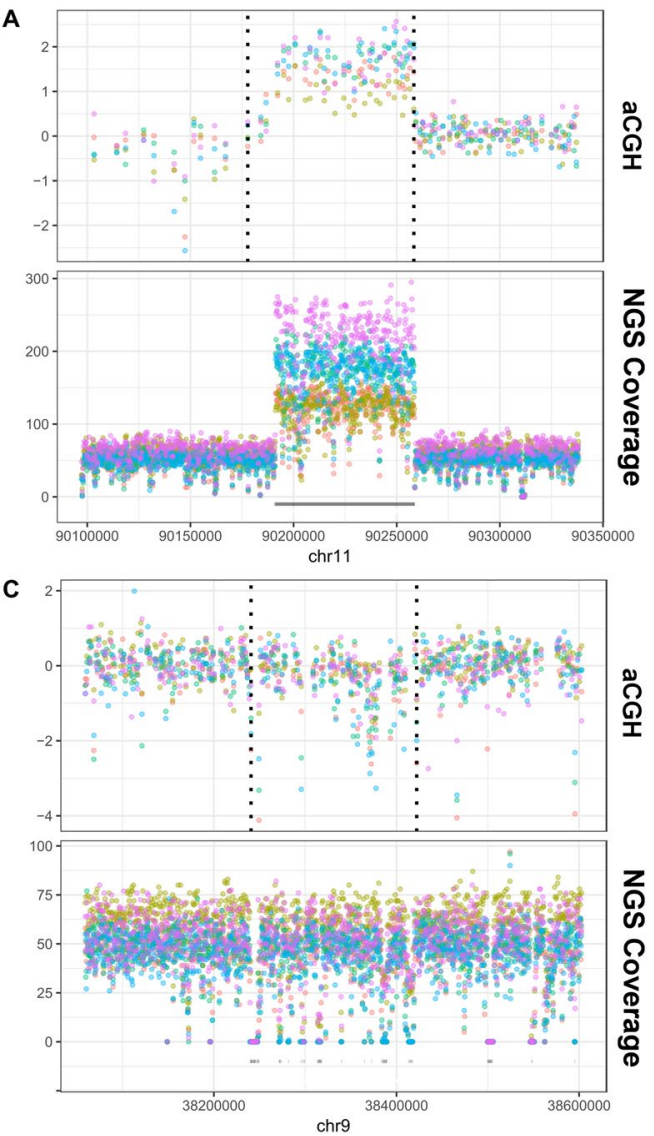

B

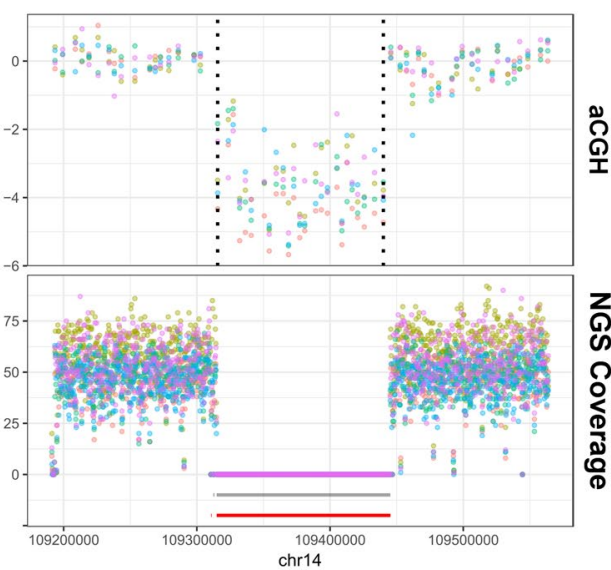

D

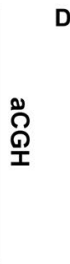

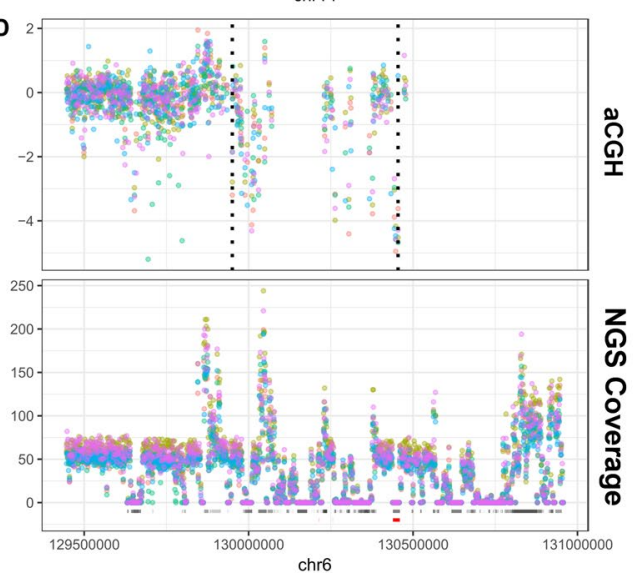

MutaMouse 1

MutaMouse 2

MutaMouse 3

MutaMouse 4

MutaMouse 5

Figure 4. Four examples of CNVs predicted using aCGH (probes shown as points in top panels; CNV call between dotted lines) overlaid with their respective NGS coverage (bottom panels; NGS coverage is shown as points; CNVnator calls from NGS data shown as black lines below coverage; Manta calls from NGS data shown as red lines below coverage). We find that some CNVs predicted using aCGH are consistent across the entire call region when compared with NGS coverage. On the other hand, many regions predicted as CNVs by aCGH represent regions of the genome encompassing many small deletions and/or gains. (A) A duplication on chromosome 11 showing congruent results between aCGH and NGS. (B) A deletion on chromosome 14 showing congruent results between aCGH and NGS. (C) A deletion on chromosome 9 predicted as one large deletion by aCGH that NGS has revealed is composed of many small deletions. (D) A deletion on chromosome 6 predicted as one large deletion by aCGH that NGS revealed is composed of many small deletions as well as several copy number gains.

\begin{abstract}
Methods
Animals sequenced and experimental design. A colony of MutaMouse animals has been maintained at HC since September 1990, producing 98 generations of animals as of December 2018. Each generation is composed of the offspring from 20 to 60 females, avoiding brother and sister matings. Maintenance of this colony and the use of animals for this study were approved by the Health Canada Ottawa Animal Care Committee. All experimental procedures, including euthanasia, were conducted in accordance with relevant guidelines and regulations. We sequenced spleen DNA from five male animals from generation 78 (April 2013) to an average base depth of 63X. We used three platforms (Illumina HiSeq, Illumina NextSeq and Pacific Biosciences) and three library insert sizes (500 bp and $3 \mathrm{~kb}$ for Illumina, $20 \mathrm{~kb}$ for Pacific Biosciences). Sequencing with paired-end reads, mate pair libraries, and long-read PacBio was undertaken to increase our sensitivity for the identification of SVs.

To compare the genetic variation between geographically separated colonies of mice, we obtained two MutaMouse animals from the Covance breeding colony in the UK. The two colonies housed at Covance and HC were bred as separate lineages since the HC colony was established in 1990 . However, the Covance colony experienced a founder effect when a few breeding pairs were used to restart the colony around 2006 (12 years prior to the collection of the animals used in this study). This colony was otherwise maintained in a manner similar to that of the HC colony.
\end{abstract}

Illumina truseq and nextera mate pair library construction and sequencing. DNA was isolated from the spleens of five MutaMouse males (designated MutaMouse 1 through 5) using the QiaQuick Blood and Tissue kit (Qiagen). TruSeq paired-end libraries were prepared for all animals by shearing $1 \mu \mathrm{g}$ of DNA with a Covaris ultrasonic disruptor followed by ligation of Illumina adapter sequences as per the manufacturer's 


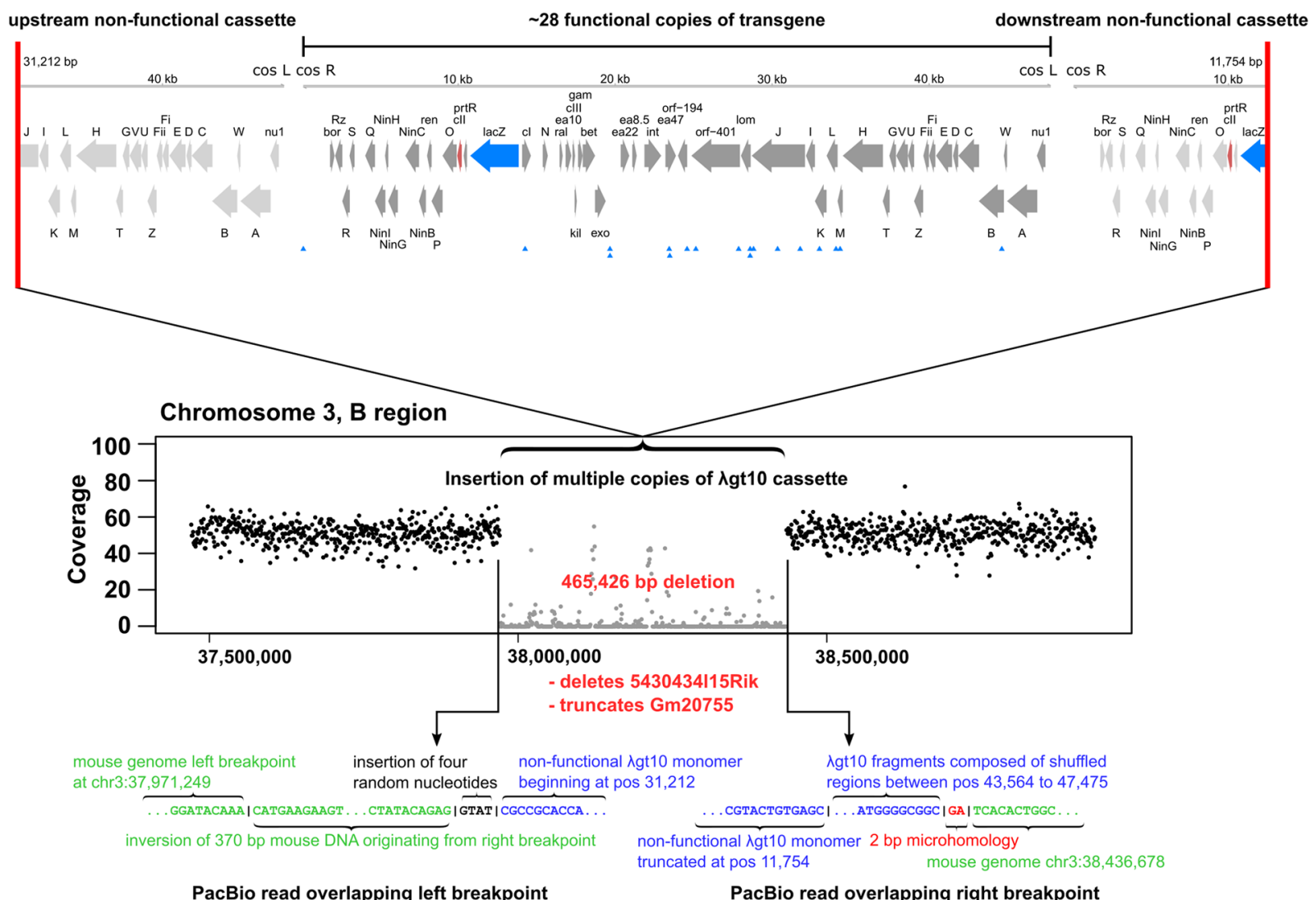

Figure 5. Assembly of $\lambda$ gt10-lacZ transgene integration site using PacBio read data and NGS coverage data. There are approximately 29 copies of the $\lambda$ gt 10 ori replication site ${ }^{35}$. However, we show here that at least one copy (at the right breakpoint, downstream of the integration site) is non-functional. There are $18 \mathrm{SNVs}$ that are constitutional in every copy of the transgene (shown by blue arrows in the top panel for one transgene monomer, and listed in Table 5). The breakpoints near the integration site were resolved by the use of long PacBio reads that map to both the $\lambda$ gt10 transgene sequence and mouse genomic sequence (bottom panel, colored text). The integration of the transgene comprised an unusual event involving (1) the deletion of $\sim 465$ Kbp of mouse genomic sequence, (2) the inversion of $370 \mathrm{bp}$ of mouse genomic sequence from the downstream breakpoint to the upstream breakpoint, (3) the insertion of two partial truncated transgene monomers at either breakpoint, and (4) the insertion of shuffled transgene sequence at the downstream breakpoint as well as the insertion of several random nucleotides at the upstream breakpoint.

protocol. DNA from MutaMouse animal 2 was additionally used to prepare a Nextera Mate Pair library. Libraries were prepared by the Génome Québec Innovation Centre (Montréal, QC).

Low-coverage sequencing of MutaMouse 1 through 5 was carried out by the Génome Québec Innovation Centre (Montreal, Canada) using one lane of a HiSeq. 2500 (PE150). An entire additional HiSeq lane was used for the Nextera Mate Pair library of MutaMouse animal 2. The sequencing depth for all animals was increased by re-sequencing the same libraries in-house on a NextSeq. 500 (using the $300 \mathrm{~V} 2$ sequencing kit) using one flow cell per animal sequenced. The two additional mice (male and female) from the Covance MutaMouse colony were used to construct Nextera Mate Pair libraries, which were then sequenced on the NextSeq. 500 as described above. All Illumina sequence reads produced were paired end, $150 \mathrm{bp}$. Data output from the sequencing are shown in Table 1.

Pacific biosciences sequencing. DNA was isolated from MutaMouse animal 2 using phenol-chloroform extraction to obtain high molecular weight fragments for long read sequencing. This DNA was processed into a SMRTbell large insert library and sequenced using 24 SMRTcells on the PacBio RSII sequencing instrument. PacBio sequencing was carried out by Génome Québec.

Variant calling. Variants were called using the Genome Analysis Toolkit (GATK version 3.6.0 $0^{52}$ best practices). No changes to the toolkit settings were implemented except for those necessary to use the mm 10 build (GRCm38) of the Mus musculus genome. The FASTQ files obtained from sequencing were aligned using BWA (version 0.7.12-r1039) using the maximal exact match (MEM) algorithm with duplicate marking enabled. The resulting BAM files were sorted using Samtools (version 1.3), then duplicates were removed using the Picard criteria and BAM files were merged per animal using Picard tools (version 2.5.0). GATK was used to perform base 


\begin{tabular}{|l|l|l|}
\hline Position & Reference Base & Alternate Base \\
\hline 137 & AG & A \\
\hline 14266 & C & CG \\
\hline 19670 & T & A \\
\hline 19673 & A & C \\
\hline 23445 & G & C \\
\hline 23481 & A & G \\
\hline 24583 & TA & T \\
\hline 25143 & A & G \\
\hline 27867 & T & C \\
\hline 28598 & G & A \\
\hline 28599 & T & A \\
\hline 28817 & A & T \\
\hline 30349 & CT & C \\
\hline 31786 & A & G \\
\hline 33016 & T & C \\
\hline 34070 & A & G \\
\hline 34331 & T & C \\
\hline 44629 & T & C,A,G \\
\hline
\end{tabular}

Table 5. Constitutional variants that are present in all copies of the MutaMouse transgene across all animals sequenced.

A

$$
\text { Origin of Homozygous }
$$$$
\text { Variants in MutaMouse }
$$
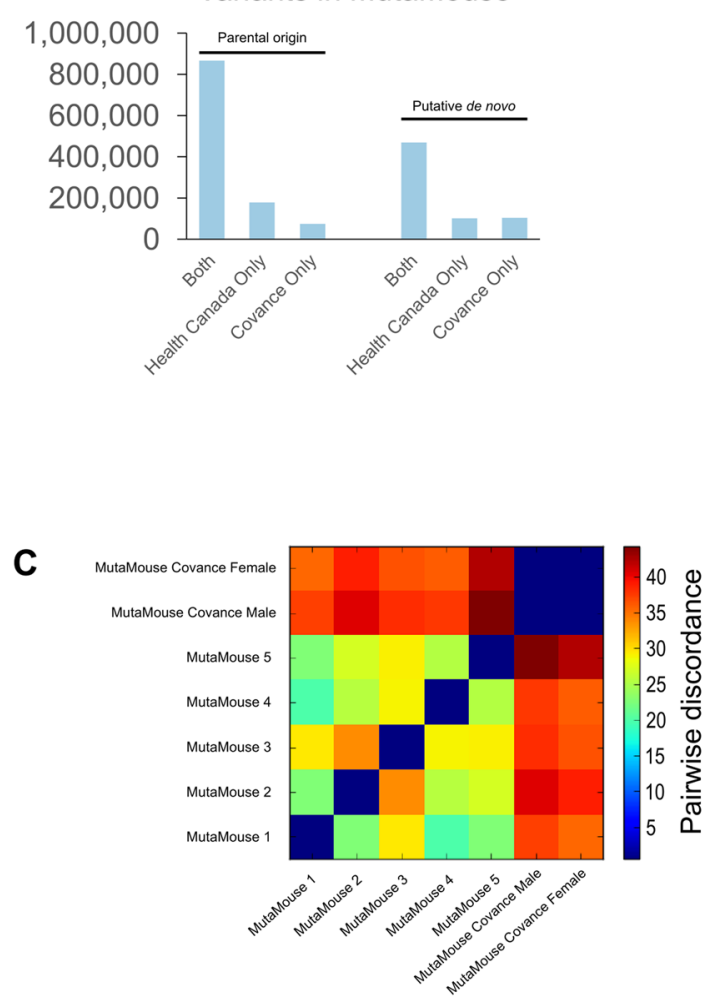

B

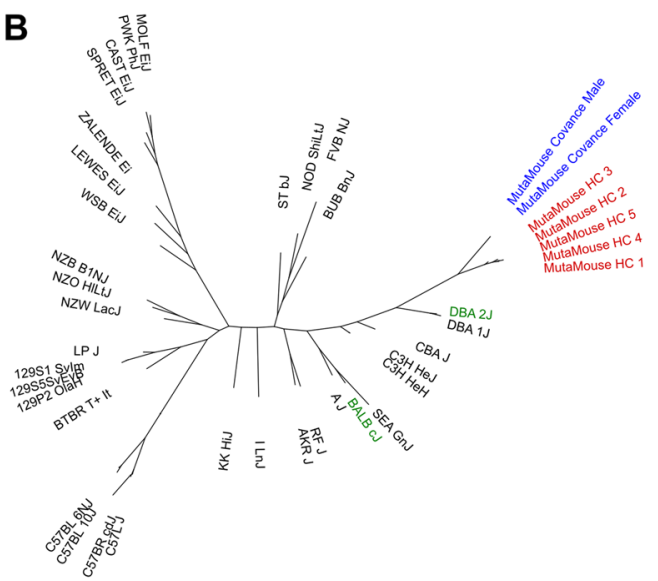

Figure 6. Comparison of variants discovered in MutaMouse colonies that were geographically isolated for 23 years of breeding (78 generations). (A) More SNVs of parental origin were retained in the Health Canada colony than the Covance colony, which experienced a population bottleneck circa 2006. The number of putative $d e$ novo SNVs is comparable, suggesting that the germline mutation rates do not differ between the populations. (B) On a phylogenetic tree, the MutaMouse colony from Covance forms a distinct branch, which is consistent with our finding that (C) Animals from the Covance MutaMouse colony have higher genotype discordance with animals from Health Canada than with each other. 
quality score recalibration and variant calling using HaplotypeCaller tool to produce gVCFs. All animals were jointly genotyped using GenotypeGVCFs, and variant quality score recalibration was performed separately for SNVs and indels using the VariantRecalibrator and ApplyRecalibration, using dbSNP for mouse v142 as the truth set of known variants. The final callset was produced by filtering variants to include $99.9 \%$ of sites in the truth set. Variants that were not marked PASS were removed from downstream analysis. Overlap between datasets was determined using BCFtools stats (version 1.3).

Runs of homozygosity (stretches of sequence for which only homozygous variant calls were made) were estimated using BCFtools roh version 1.3.

We compiled a list of $642,1434,408$ and 45 genes involved in cancer progression, cell cycle regulation, DNA repair, and 45 xenobiotic metabolism, respectively. Cancer genes were identified from the COSMIC database, accessed Sept 11,2016, while genes in the other pathways were identified by their gene ontology [GO] terms (Supplemental Table S2). We used this list to extract MutaMouse variants that are present in coding sequences for genes involved in toxicological functions.

Structural variant calling. SVs including duplication or translocation breakpoints, small insertions and deletions $(<50 \mathrm{bp})$, as well as large insertions and deletions ( $>50 \mathrm{bp}$ ), were identified using Manta (version 1.01; Illumina; http://github.com/Illumina/manta). This software relies on the identification of discordant paired-end reads that would normally exist in the forward-reverse (FR) orientation. Different types of structural rearrangements can be inferred by observed alignments (e.g., RR orientation indicates an inverted DNA sequence ${ }^{48}$ ). Mate pair libraries were also converted to pseudo-paired-end format by reversing the SAM flags $0 \times 16$ and $0 \times 32$ for each read, thereby converting the normal reads to the conventional FR orientation and enabling the use of Manta for mate-pair libraries. The resulting VCF files for called diploid SVs were filtered stringently using the suggested Manta filters. Furthermore, split read support for the alternate allele had to achieve a minimum sequencing depth of 20 and consist of precisely determined breakpoints.

Copy number variant analysis using array comparative genomic hybridization and NGS. Genomic DNA was extracted from C57BL/6 spleen obtained from Jackson Laboratory (stock 000664) using the QiaQuick Blood and Tissue kit (Qiagen, Valencia, CA, USA). For each MutaMouse animal analyzed, $1 \mu \mathrm{g}$ MutaMouse genomic DNA and $1 \mu \mathrm{g}$ C57BL/6 reference DNA was labeled with Cy5 and Cy3, respectively (5191-3400 SureTag DNA Labelling Kit; Agilent Technologies, Cedar Creek, TX, USA). For each sample, labelled DNA was mixed and hybridized to an Agilent SurePrint G3 Mouse CGH array with $1 \mathrm{M}$ probes (G4838A; Agilent Technologies) according to the manufacturer's directions (Agilent Oligonucleotide Array-Based CGH for Genomic DNA Analysis; version 7.3). Slides were scanned using an Agilent scanner and data extracted using Agilent Feature Extraction Software v10.5. Signal intensities from Cy3 and Cy5 were then background subtracted and subjected to Lowess normalization using Rank invariant probes. Probes that were identified as outliers (i.e., affected by their position on the array) were eliminated from the dataset. CNVs were called from the probe values using R-Gada ${ }^{53}$.

CNVs were called from NGS data using CNVnator version $0.3 .3^{47}$ and filtered based on the $\mathrm{t}$-test $(\mathrm{p}<0.05)$. A bin size of $250 \mathrm{bp}$ was used for read partitioning for CNV calling. The overlap between datasets consisting of aCGH, CNVnator, and Manta calls was determined using bedtools v2.26.0. Genetic variation (SNVs and CNVs) between the five MutaMouse animals from a colony maintained at HC is reported graphically using $\operatorname{circos}_{\text {plot }}{ }^{54}$.

Transgene breakpoint characterization, de novo assembly, and variants. We attempted to identify the location of the breakpoints using a published method ${ }^{55}$. However, the aligned reads were located on many different chromosomes with no obvious pattern, suggesting that transgene integration took place in a genomic region containing repetitive elements. Therefore, we used the long read lengths produced by PacBio sequencing to first align the reads to the $\lambda$ gt10 sequence ${ }^{35}$ using BWA-MEM with the -x pacbio flag enabled (equivalent to enabling the following settings: -k17 -W40 -r10 -A1 -B1 -O1 -E1 -L0). Subsequently, we aligned the subset of reads with sequence similarity to $\lambda$ gt10 back to the mouse genome. This resulted in the identification of reads containing regions of overlap with both mouse genomic DNA and $\lambda$ gt10 sequences, thereby identifying the breakpoints.

Shotgun sequence Illumina reads from MutaMouse animal 2 were aligned to the $\lambda$ gt 10 reference sequence using BWA-MEM as described for variant calling. Aligned reads were extracted using Samtools v1.3 and assembled using SPAdes version 3.9.1. A 47,588 bp contig with 809X coverage was obtained and used for downstream analysis. Reads were re-aligned to this new reference contig using the same procedure, and variants were called using GATK HaplotypeCaller using a ploidy of 31 (i.e., 1 out of $\sim 29$ copies of the transgene in the MutaMouse should still be above the detection limit). This contig was annotated using Prokka v1.12, using default settings and the-proteins flag set to use the $\lambda$ phage reference sequence NC_001416. The genes were visualized using the R package Gviz ${ }^{56}$.

Comparative genomics analysis. The number of homozygous variants for each mouse from the HC and Covance colony was enumerated using BCFtools. SNPhylo ${ }^{57}$ was used to build a phylogenetic tree from a multi-sample VCF file. The pairwise genotype concordance for each mouse was calculated using BCFtools gtcheck. The Ensembl Variant Effect Predictor version $89.7^{58}$ and SnpEff version $4.1 \mathrm{k}^{59}$ was used to predict the consequences of variants and create annotated VCF files. For comparison to parental strains DBA/2 J and BALB/ cJ, we used the strain-specific SNPs and indels found in dbSNP v142.

Data access. The raw sequences generated for this study are available in the SRA under BioProject No. PRJNA471839. 


\section{References}

1. Zeiger, E. Historical perspective on the development of the genetic toxicity test battery in the United States. Environ Mol Mutagen 51, 781-791, https://doi.org/10.1002/em.20602 (2010).

2. Eastmond, D. A. et al. Mutagenicity testing for chemical risk assessment: update of the WHO/IPCS Harmonized Scheme. Mutagenesis 24, 341-349, https://doi.org/10.1093/mutage/gep014 (2009).

3. Lambert, I. B., Singer, T. M., Boucher, S. E. \& Douglas, G. R. Detailed review of transgenic rodent mutation assays. Mutat Res 590, 1-280, https://doi.org/10.1016/j.mrrev.2005.04.002 (2005).

4. OECD. Test 488: Transgenic Rodent Somatic and Germ Cells Gene Mutation Assays. Vol. Section 4 (OECD Publishing, 2013).

5. Marchetti, F. et al. Identifying germ cell mutagens using OECD test guideline 488 (transgenic rodent somatic and germ cell gene mutation assays) and integration with somatic cell testing. Mutat Res 832-833, 7-18, https://doi.org/10.1016/j.mrgentox.2018.05.021 (2018).

6. O’Brien, J. M. et al. Transgenic rodent assay for quantifying male germ cell mutant frequency. J Vis Exp, e51576, https://doi. org/10.3791/51576 (2014).

7. Gingerich, J. D., Soper, L., Lemieux, C. L., Marchetti, F. \& Douglas, G. R. Transgenic Rodent Gene Mutation Assay in Somatic Tissues. (Springer Science + Business Media, 2014).

8. Beal, M. A., Gagne, R., Williams, A., Marchetti, F. \& Yauk, C. L. Characterizing Benzo[a]pyrene-induced lacZ mutation spectrum in transgenic mice using next-generation sequencing. BMC Genomics 16, 812, https://doi.org/10.1186/s12864-015-2004-4 (2015).

9. Besaratinia, A. et al. A high-throughput next-generation sequencing-based method for detecting the mutational fingerprint of carcinogens. Nucleic Acids Res 40, e116, https://doi.org/10.1093/nar/gks610 (2012).

10. Labib, S. et al. A framework for the use of single-chemical transcriptomics data in predicting the hazards associated with complex mixtures of polycyclic aromatic hydrocarbons. Arch Toxicol 91, 2599-2616, https://doi.org/10.1007/s00204-016-1891-8 (2017).

11. Chepelev, N. L. et al. Transcriptional profiling of the mouse hippocampus supports an NMDAR-mediated neurotoxic mode of action for benzo[a]pyrene. Environ Mol Mutagen 57, 350-363, https://doi.org/10.1002/em.22020 (2016).

12. Chepelev, N. L. et al. Transcriptional Profiling of Dibenzo[def,p]chrysene-induced Spleen Atrophy Provides Mechanistic Insights into its Immunotoxicity in MutaMouse. Toxicol Sci 149, 251-268, https://doi.org/10.1093/toxsci/kfv232 (2016).

13. Moffat, I. et al. Comparison of toxicogenomics and traditional approaches to inform mode of action and points of departure in human health risk assessment of benzo[a]pyrene in drinking water. Crit Rev Toxicol 45, 1-43, https://doi.org/10.3109/10408444.20 14.973934 (2015)

14. Uchimura, A. et al. Germline mutation rates and the long-term phenotypic effects of mutation accumulation in wild-type laboratory mice and mutator mice. Genome Res 25, 1125-1134, https://doi.org/10.1101/gr.186148.114 (2015).

15. Ardin, M. et al. MutSpec: a Galaxy toolbox for streamlined analyses of somatic mutation spectra in human and mouse cancer genomes. BMC Bioinformatics 17, 170, https://doi.org/10.1186/s12859-016-1011-z (2016).

16. Locke, M. E. et al. Genomic copy number variation in Mus musculus. BMC Genomics 16, 497, https://doi.org/10.1186/s12864-0151713-z (2015).

17. Nik-Zainal, S. et al. The genome as a record of environmental exposure. Mutagenesis 30, 763-770, https://doi.org/10.1093/mutage/ gev073 (2015).

18. Mudge, J. M. \& Harrow, J. Creating reference gene annotation for the mouse C57BL6/J genome assembly. Mamm Genome 26, 366-378, https://doi.org/10.1007/s00335-015-9583-x (2015).

19. Yalcin, B., Adams, D. J., Flint, J. \& Keane, T. M. Next-generation sequencing of experimental mouse strains. Mamm Genome 23, 490-498, https://doi.org/10.1007/s00335-012-9402-6 (2012).

20. Yalcin, B. et al. The fine-scale architecture of structural variants in 17 mouse genomes. Genome Biol 13, R18, https://doi.org/10.1186/ gb-2012-13-3-r18 (2012).

21. Wong, K. et al. Sequencing and characterization of the FVB/NJ mouse genome. Genome Biol 13, R72, https://doi.org/10.1186/gb2012-13-8-r72 (2012)

22. Yalcin, B. et al. Sequence-based characterization of structural variation in the mouse genome. Nature 477, 326-329, https://doi. org/10.1038/nature10432 (2011).

23. Keane, T. M. et al. Mouse genomic variation and its effect on phenotypes and gene regulation. Nature 477, 289-294, https://doi. org/10.1038/nature10413 (2011).

24. Agam, A. et al. Elusive copy number variation in the mouse genome. PLoS One 5, e12839, https://doi.org/10.1371/journal. pone.0012839 (2010).

25. Adams, D. J., Doran, A. G., Lilue, J. \& Keane, T. M. The Mouse Genomes Project: a repository of inbred laboratory mouse strain genomes. Mamm Genome 26, 403-412, https://doi.org/10.1007/s00335-015-9579-6 (2015).

26. Lee, Y. K. et al. Unique profile of ordered arrangements of repetitive elements in the C57BL/6J mouse genome implicating their functional roles. PLoS One 7, e35156, https://doi.org/10.1371/journal.pone.0035156 (2012).

27. Nguyen, C., Baten, A. \& Morahan, G. Comparison of sequence variants in transcriptomic control regions across 17 mouse genomes. Database (Oxford) 2014, bau020, https://doi.org/10.1093/database/bau020 (2014).

28. Sarsani, V. K. et al. The Genome of C57BL/6J "Eve", the Mother of the Laboratory Mouse Genome Reference Strain. G3 (Bethesda), https://doi.org/10.1534/g3.119.400071 (2019).

29. Eppig, J. T. et al. Mouse Genome Informatics (MGI): reflecting on 25 years. Mamm Genome 26, 272-284, https://doi.org/10.1007/ s00335-015-9589-4 (2015).

30. Simon, M. M. et al. A comparative phenotypic and genomic analysis of C57BL/6J and C57BL/6N mouse strains. Genome Biol 14, R82, https://doi.org/10.1186/gb-2013-14-7-r82 (2013).

31. Ananda, G., Takemon, Y., Hinerfeld, D. \& Korstanje, R. Whole-genome sequence of the C57L/J mouse inbred strain. G3 (Bethesda) 4, 1689-1692, https://doi.org/10.1534/g3.114.012997 (2014).

32. Buchner, D. A. \& Nadeau, J. H. Contrasting genetic architectures in different mouse reference populations used for studying complex traits. Genome Res 25, 775-791, https://doi.org/10.1101/gr.187450.114 (2015).

33. Doran, A. G. et al. Deep genome sequencing and variation analysis of 13 inbred mouse strains defines candidate phenotypic alleles, private variation and homozygous truncating mutations. Genome Biol 17, 167, https://doi.org/10.1186/s13059-016-1024-y (2016).

34. Kumar, P., Henikoff, S. \& Ng, P. C. Predicting the effects of coding non-synonymous variants on protein function using the SIFT algorithm. Nat Protoc 4, 1073-1081, https://doi.org/10.1038/nprot.2009.86 (2009).

35. Shwed, P. S., Crosthwait, J., Douglas, G. R. \& Seligy, V. L. Characterisation of MutaMouse lambdagt10-lacZ transgene: evidence for in vivo rearrangements. Mutagenesis 25, 609-616, https://doi.org/10.1093/mutage/geq.048 (2010).

36. Blakey, D. H., Douglas, G. R., Huang, K. C. \& Winter, H. J. Cytogenetic mapping of lambda gt10 lacZ sequences in the transgenic mouse strain 40.6 (Muta Mouse). Mutagenesis 10, 145-148 (1995).

37. Tsang, S. et al. A comprehensive SNP-based genetic analysis of inbred mouse strains. Mamm Genome 16, 476-480, https://doi. org/10.1007/s00335-005-0001-7 (2005).

38. Rau, C. D. et al. High-Density Genotypes of Inbred Mouse Strains: Improved Power and Precision of Association Mapping. G3 (Bethesda) 5, 2021-2026, https://doi.org/10.1534/g3.115.020784 (2015).

39. Takada, T., Yoshiki, A., Obata, Y., Yamazaki, Y. \& Shiroishi, T. NIG_MoG: a mouse genome navigator for exploring intersubspecific genetic polymorphisms. Mamm Genome 26, 331-337, https://doi.org/10.1007/s00335-015-9569-8 (2015). 
40. Oey, H., Isbel, L., Hickey, P., Ebaid, B. \& Whitelaw, E. Genetic and epigenetic variation among inbred mouse littermates: identification of inter-individual differentially methylated regions. Epigenetics Chromatin 8, 54, https://doi.org/10.1186/s13072-0150047-z (2015).

41. Safe, S., Lee, S. O. \& Jin, U. H. Role of the aryl hydrocarbon receptor in carcinogenesis and potential as a drug target. Toxicol Sci 135, 1-16, https://doi.org/10.1093/toxsci/kft128 (2013).

42. Lemieux, C. L. et al. Simultaneous measurement of benzo[a]pyrene-induced Pig-a and lacZ mutations, micronuclei and DNA adducts in Muta Mouse. Environ Mol Mutagen 52, 756-765, https://doi.org/10.1002/em.20688 (2011).

43. Miosge, L. A. et al. Comparison of predicted and actual consequences of missense mutations. Proc Natl Acad Sci USA 112, E5189-5198, https://doi.org/10.1073/pnas.1511585112 (2015).

44. Labib, S. et al. Subchronic oral exposure to benzo(a)pyrene leads to distinct transcriptomic changes in the lungs that are related to carcinogenesis. Toxicol Sci 129, 213-224, https://doi.org/10.1093/toxsci/kfs177 (2012).

45. Malik, A. I., Williams, A., Lemieux, C. L., White, P. A. \& Yauk, C. L. Hepatic mRNA, microRNA, and miR-34a-target responses in mice after 28 days exposure to doses of benzo(a)pyrene that elicit DNA damage and mutation. Environ Mol Mutagen 53, 10-21, https://doi.org/10.1002/em.20668 (2012).

46. Sudmant, P. H. et al. An integrated map of structural variation in 2,504 human genomes. Nature 526, 75-81, https://doi.org/10.1038/ nature15394 (2015).

47. Abyzov, A., Urban, A. E., Snyder, M. \& Gerstein, M. CNVnator: an approach to discover, genotype, and characterize typical and atypical CNVs from family and population genome sequencing. Genome Res 21, 974-984, https://doi.org/10.1101/gr.114876.110 (2011).

48. Chen, X. et al. Manta: rapid detection of structural variants and indels for germline and cancer sequencing applications. Bioinformatics 32, 1220-1222, https://doi.org/10.1093/bioinformatics/btv710 (2016).

49. Liu, P. et al. Chromosome catastrophes involve replication mechanisms generating complex genomic rearrangements. Cell 146, 889-903, https://doi.org/10.1016/j.cell.2011.07.042 (2011).

50. Hastings, P. J., Ira, G. \& Lupski, J. R. A microhomology-mediated break-induced replication model for the origin of human copy number variation. PLoS Genet 5, e1000327, https://doi.org/10.1371/journal.pgen.1000327 (2009).

51. Carvalho, C. M. \& Lupski, J. R. Mechanisms underlying structural variant formation in genomic disorders. Nat Rev Genet 17, 224-238, https://doi.org/10.1038/nrg.2015.25 (2016).

52. DePristo, M. A. et al. A framework for variation discovery and genotyping using next-generation DNA sequencing data. Nat Genet 43, 491-498, https://doi.org/10.1038/ng.806 (2011).

53. Pique-Regi, R., Caceres, A. \& Gonzalez, J. R. R-Gada: a fast and flexible pipeline for copy number analysis in association studies. BMC Bioinformatics 11, 380, https://doi.org/10.1186/1471-2105-11-380 (2010).

54. Krzywinski, M. et al. Circos: an information aesthetic for comparative genomics. Genome Res 19, 1639-1645, https://doi. org/10.1101/gr.092759.109 (2009)

55. Masumura, K. et al. Estimation of the frequency of inherited germline mutations by whole exome sequencing in ethyl nitrosoureatreated and untreated gpt delta mice. Genes Environ 38, 10, https://doi.org/10.1186/s41021-016-0035-y (2016).

56. Hahne, F. \& Ivanek, R. In Statistical Genomics. Methods in Molecular Biology Vol. 1418 (eds Mathe', E. \& David, S.) (Humana Press, 2016).

57. Lee, T. H., Guo, H., Wang, X., Kim, C. \& Paterson, A. H. SNPhylo: a pipeline to construct a phylogenetic tree from huge SNP data. BMC Genomics 15, 162, https://doi.org/10.1186/1471-2164-15-162 (2014).

58. McLaren, W. et al. The Ensembl Variant Effect Predictor. Genome Biol 17, 122, https://doi.org/10.1186/s13059-016-0974-4 (2016).

59. Cingolani, P. et al. A program for annotating and predicting the effects of single nucleotide polymorphisms, SnpEff: SNPs in the genome of Drosophila melanogaster strain w1118; iso-2; iso-3. Fly (Austin) 6, 80-92, https://doi.org/10.4161/fly.19695 (2012).

\section{Acknowledgements}

This work is supported by funding through Health Canada's Genomics Research and Development Initiative. We thank Carol Beevers and Julie Clements for providing the animals from the MutaMouse colony maintained at Covance.

\section{Author Contributions}

M.M. with help from M.A.B. sequenced the MutaMouse genome, contributed to data analysis and interpretation. A.S. performed data analyses on the effect of read depth and library preparation on variant calling. C.Y. and F.M. secured the funding for the study and were responsible for study conception, study design and results interpretation. All authors contributed to manuscript writing and approved the final version.

\section{Additional Information}

Supplementary information accompanies this paper at https://doi.org/10.1038/s41598-019-50302-0.

Competing Interests: The authors declare no competing interests.

Publisher's note Springer Nature remains neutral with regard to jurisdictional claims in published maps and institutional affiliations.

(c) (i) Open Access This article is licensed under a Creative Commons Attribution 4.0 International cc. License, which permits use, sharing, adaptation, distribution and reproduction in any medium or format, as long as you give appropriate credit to the original author(s) and the source, provide a link to the Creative Commons license, and indicate if changes were made. The images or other third party material in this article are included in the article's Creative Commons license, unless indicated otherwise in a credit line to the material. If material is not included in the article's Creative Commons license and your intended use is not permitted by statutory regulation or exceeds the permitted use, you will need to obtain permission directly from the copyright holder. To view a copy of this license, visit http://creativecommons.org/licenses/by/4.0/.

(c) The Author(s) 2019 